\title{
NEW VOLUME COMPARISON RESULTS AND APPLICATIONS TO DEGENERATION OF RIEMANNIAN METRICS
}

\author{
QI S. ZHANG AND MENG ZHU
}

\begin{abstract}
We consider a condition on the Ricci curvature involving vector fields, which is broader than the Bakry-Émery Ricci condition. Under this condition volume comparison, Laplacian comparison, isoperimetric inequality and gradient bounds are proven on the manifold.

Specializing to the Bakry-Émery Ricci curvature condition, we initiate an approach to work on the original manifold, which yields, under a weaker than usual assumption, the results mentioned above for the original manifold. These results are different from most well known ones in the literature where the conclusions are made on the weighted manifold instead.

Applications on convergence and degeneration of Riemannian metrics under this curvature condition are given. To this effect, in particular for the Bakry-Émery Ricci curvature condition, the gradient of the potential function is allowed to have singularity of order close to 1 while the traditional method of weighted manifolds allows bounded gradient. This approach enables us to extend some of the results in the papers $\mathrm{Co}$, [ChCo2, [ZZ, [TZ and [WZ]. The condition also covers general Ricci solitons instead of just gradient Ricci solitons.
\end{abstract}

\section{Contents}

1. Introduction

2. New volume comparison theorems

2.1. Ricci vector field condition

2.2. Bakry-Émery Ricci condition

3. Local isoperimetric and sobolev constants estimates

4. Gradient estimates, mean value inequality, heat kernel bounds and cut-off functions

5. Segment inequality, excess estimate and $\epsilon$-splitting map

6. Main theorems: volume continuity, metric cone, size of singular set 30

7. Appendix I

8. Appendix II

Acknowledgements 44

References

\section{INTRODUCTION}

The volume comparison theorem by Bishop-Gromov is an essential tool in differential geometry and analysis on manifolds. It states that if the Ricci curvature is bounded from below by a constant, the ratio between the volume of a geodesic ball and the volume of the ball of the same radius in the model space is a non-increasing function of the radius. 
However, in many situations, the point-wise lower bound on the Ricci curvature is not available. Hence many efforts are made to relax this assumption. In the paper [PeWe1, Petersen and Wei obtained a bound on the above volume ratio under the assumption that the norm of the negative part of the Ricci curvature is an $L^{p}$ function with $p>n / 2$, where $n$ is the dimension of the manifold. The bound approaches 1 when the radii of the balls converge to 0. Another successful approach of relaxing the Ricci lower bound condition is to consider a lower bound of the Bakry-Émery Ricci curvature ([BE] $)$. A typical condition is

$$
\operatorname{Ric}+\operatorname{Hess} L \geq-\lambda g
$$

for a nonnegative constant $\lambda$ and a smooth function $L$ such that $L$ and/or $|\nabla L|$ is bounded. Under this condition, for the weighted manifold $\left(\mathbf{M}, d v o l=e^{-L} d g\right)$, several authors derived Laplacian comparison theorems for the weighted Laplacian $\Delta-\nabla L \cdot \nabla$ and/or bounds on the volume ratio of weighted balls. See Qian [Q], Bakry and Qian [BQ], X.D. $\mathrm{Li}$ XLi and Wei and Wylie WW. All of these results have led to many applications which can be found in the forward references in MathSciNet etc. Their results also yield certain bounds on volumes of geodesic balls on the original manifold involving $\sup L$ and $\inf L$, or $\nabla L$. However, monotonicity or almost monotonicity of volume ratios on the original geodesic balls do not follow directly in general.

In this paper, we consider a Ricci curvature condition involving vector fields, which is broader than the Bakry-Émery Ricci curvature condition (1.1). It is also more general than curvature dimension inequality. More specifically, we impose a lower bound condition on a modified Ricci curvature

$$
\operatorname{Ric}+\frac{1}{2} \mathcal{L}_{V} g
$$

where $V$ is a smooth vector field, and $\mathcal{L}_{V} g$ is the Lie derivative of $g$ in the direction of $V$. In local coordinates, (1.2) can be written as

$$
R_{i j}+\frac{1}{2}\left(\nabla_{i} V_{j}+\nabla_{j} V_{i}\right)
$$

where $V=V^{i} \partial_{i}$ and $V_{i}=g_{i j} V^{j}$. If $V=\nabla L$ for a smooth function $L$, then this is nothing but the Bakry-Émery Ricci curvature. The study of Ricci solitons and Einstein field equation provide strong motivation for considering this kind of Ricci condition. Let us recall that a Riemannian manifold $(\mathbf{M}, g)$ is called a Ricci soliton if there exists a smooth vector field $V$ and a constant $\lambda$ such that

$$
\operatorname{Ric}+\frac{1}{2} \mathcal{L}_{V} g+\lambda g=0
$$

Also Einstein's field equation can be expressed as

$$
R_{i j}-\frac{1}{2} R g_{i j}+\lambda g_{i j}=\frac{8 \pi G}{c^{4}} T_{i j}
$$

where $R$ is the scalar curvature, $G$ is Newton's gravitational constant and $c$ is the speed of light in vacuum. $T_{i j}$ is the stress-energy tensor which may take the special form $\nabla_{i} V_{j}+\nabla_{j} V_{i}$ or $\nabla^{2} L$. Another motivation comes from the study of the so called modified Ricci flows where the Ricci curvature is modified by a vector field in the same manner as here. In addition, this kind of conditions have been used in other context such as [D], [FG] and [W] to obtain diameter bound or bounds on fundamental groups for manifolds . 
An underlining feature of the paper is that our emphasis is on the original manifolds and the standard Laplacian. By assuming the modified Ricci curvature (1.2) having a lower bound, volume comparison, Laplacian comparison and gradient bounds are proven for the manifold. This approach has the advantage that even for the Bakry-Émery Ricci curvature, i.e, $V=\nabla L$, it can be shown that under a weaker than usual condition on the potential function that much of the volume ratio bounds, Laplacian comparison theorem and gradient bounds still hold for the original manifolds. For instance, the results here provide almost monotonicity of volume ratios in the spirit of Bishop-Gromov volume comparison on the original manifold without the boundedness of the $|\nabla L|$. These properties are necessary for proving convergence results on general manifolds, Ricci solitons and other applications which will be presented later in the paper. Here are samples of the main theorems.

Theorem 1.1 (Tangent cones are metric cones, Theorem 6.6 below). Let $\left(\mathbf{M}_{i}, g_{i}\right)$ be a sequence of Riemannian manifolds satisfying (5.1) and (5.2) for some $\lambda \geq 0, K \geq 0, \alpha \in$ $[0,1)$, and $\rho>0$ and some smooth vector field $V_{i}$, i.e.,

$$
\operatorname{Ric}_{g_{i}}+\frac{1}{2} \mathcal{L}_{V_{i}} g_{i} \geq-\lambda g_{i} ; \quad\left|V_{i}\right|(\cdot) \leq \frac{K}{d_{i}\left(\cdot, O_{i}\right)^{\alpha}}, \text { for some } O_{i} \in \mathbf{M}_{i} ; \operatorname{Vol}\left(B_{g_{i}}(\cdot, 1)\right) \geq \rho \text {. }
$$

Suppose that $\left(\mathbf{M}_{i}, d_{i}\right) \stackrel{d_{G H}}{\longrightarrow}(Y, d)$ in Gromov-Hausdorff topology. Then any tangent cone of $Y$ is a metric cone. In particular, any tangent cone of a single manifold satisfying the above condition is a metric cone.

Theorem 1.2 (Size estimate of the singular set, Theorem 6.7 below). Let $\left(\mathbf{M}_{i}, g_{i}\right)$ be a sequence of Riemannian manifolds satisfying (5.1) and (5.2) for some $\lambda, K, \alpha$, and $\rho$.

Suppose that $\left(\mathbf{M}_{i}, d_{i}\right) \stackrel{d_{G H}}{\longrightarrow}(Y, d)$ in Gromov-Hausdorff topology. Then $\operatorname{dim} S(Y) \leq n-2$. Here, $S(Y)$ is the singular set of $Y$ and dim stands for the Hausdorff dimension.

The following is an outline of the proof. We first prove the volume and Laplacian comparison results under the Ricci vector field condition. Then we will move according to the road map provided in the papers [ChCo1] and [ChCo2. Namely, we need to take a number of intermediate steps of proving gradient bounds for harmonic functions, Sobolev inequality, existence of good cut off functions, segment inequality, excess estimate, barrier functions and existence of $\epsilon$-splitting maps. With the exception of gradient bound and segment inequality, the proofs of these results will involve new ingredients drawing from recent literature, which are needed since many of the model functions used in comparison arguments are not available here. The main assumption on the vector field $V$ in this paper is $|V(\cdot)| \leq \frac{K}{d(\cdot, O)^{\alpha}}$, for some $O \in \mathbf{M}$ and $\alpha \in[0,1)$. If $\alpha=0$, i.e., the vector field $V$ is bounded, then the proof can be shortened considerably. Some results can also be strengthened.

\section{New volume COMPARISON TheOREMS}

2.1. Ricci vector field condition. Let $(\mathbf{M}, g)$ be an $n$ dimensional Riemannian manifold with a fixed base point $O \in \mathbf{M}$. In this subsection, our basic assumption on the Ricci curvature tensor is

$$
\operatorname{Ric}+\frac{1}{2} \mathcal{L}_{V} g \geq-\lambda g
$$


for some constant $\lambda \geq 0$ and smooth vector field $V$ which satisfies the following condition:

$$
|V|(y) \leq \frac{K}{d(y, O)^{\alpha}}
$$

for any $y \in \mathbf{M}$. Here $d(y, O)$ represents the distance from $O$ to $y$, and $K \geq 0$ and $0 \leq \alpha<1$ are constants. Intuitively, the basic assumption (2.2) on the vector field $V$ is that it has singularity of order less than 1. Our assumptions include the popular special cases such as (1.1), i.e., Bakry-Émery Ricci curvature Ric + Hess $L$ being bounded from below, with bounded $|\nabla L|$, and gradient Ricci solitons Ric + Hess $L=\lambda g$ with just bounded potential $L$. In both of these special cases, we have $V=\nabla L$. Moreover, due to the special structure of gradient Ricci solitons, local boundedness on the potential function $L$ automatically implies local bounds on its gradient.

Let us present the Laplacian comparison theorem on which the paper is based. The proof replies on a simple but key observation that the Lie derivative or Hessian become ordinary derivative or second derivative in the radial direction.

We should mention a related result by Qian Q who long time ago obtained a Laplacian comparison theorem under a Ricci curvature condition that is modified by a vector field:

$$
\operatorname{Ric}+\frac{1}{2} \mathcal{L}_{V} g-\frac{1}{N-n} V \otimes V \geq-\lambda g .
$$

Here $V$ is a vector field, $n$ is the dimension of the manifold and $N$ is a number strictly greater than $n$. However, the result depends on $N$ which can not be taken as $n$, and the Laplace operator there is also modified accordingly. In the following, we will use $C\left(a_{1}, a_{2}, \cdots, a_{k}\right)$ to denote constants depending on parameters $a_{1}, a_{2}, \cdots, a_{k}$.

Proposition 2.1 (Laplacian comparison). Assume that (2.1) and (2.2) hold. Let $s=$ $d(y, x)$ be the distance from any point $y$ to some fixed point $x$, and $\gamma:[0, s] \rightarrow \mathbf{M} a$ normal minimal geodesic with $\gamma(0)=x$ and $\gamma(s)=y$. Then in the distribution sense,

$$
\Delta s-\frac{n-1}{s} \leq \frac{\lambda}{3} s+<V, \nabla s>+\frac{C(\alpha) K}{s^{\alpha}} .
$$

Proof. We may just prove the inequality at smooth points of $s$, since (2.3) can then be established in the distribution sense by the fact that the complement of the cut locus is star shaped.

From the Bochner formula, we have

$$
0=\frac{1}{2} \Delta|\nabla s|^{2}=\left|\nabla^{2} s\right|^{2}+<\nabla \Delta s, \nabla s>+\operatorname{Ric}(\nabla s, \nabla s) .
$$

By using Cauchy-Schwarz inequality $\left|\nabla^{2} s\right|^{2} \geq \frac{(\Delta s)^{2}}{n-1}$, it yields

$$
\frac{\partial}{\partial s} \Delta s+\frac{(\Delta s)^{2}}{n-1} \leq-\operatorname{Ric}(\nabla s, \nabla s)
$$

which is equivalent to

$$
\frac{1}{s^{2}} \frac{\partial}{\partial s}\left(s^{2} \Delta s\right)+\frac{1}{n-1}\left(\Delta s-\frac{n-1}{s}\right)^{2} \leq \frac{n-1}{s^{2}}-\operatorname{Ric}(\nabla s, \nabla s),
$$


due to the fact that

$$
\begin{aligned}
\frac{\partial}{\partial s} \Delta s+\frac{(\Delta s)^{2}}{n-1} & =\frac{\partial}{\partial s} \Delta s+\frac{(\Delta s)^{2}}{n-1}-\frac{2}{s} \Delta s+\frac{n-1}{s^{2}}+\frac{2}{s} \Delta s-\frac{n-1}{s^{2}} \\
& =\frac{1}{s^{2}} \frac{\partial}{\partial s}\left(s^{2} \Delta s\right)-\frac{n-1}{s^{2}}+\frac{1}{n-1}\left(\Delta s-\frac{n-1}{s}\right)^{2} .
\end{aligned}
$$

Multiplying both sides of (2.4) by $s^{2}$ and integrating from 0 to $s$, we get

$$
\Delta s \leq \frac{n-1}{s}-\frac{1}{s^{2}} \int_{0}^{s} t^{2} \operatorname{Ric}\left(\gamma^{\prime}(t), \gamma^{\prime}(t)\right) d t .
$$

At any point $\gamma(t)$, we may choose an orthonormal frame $\left\{e_{1}, e_{2}, \cdots, e_{n}\right\}$ with $e_{1}=\gamma^{\prime}(t)$. Then from assumption (2.1), we observe that

$$
\begin{aligned}
\operatorname{Ric}\left(\gamma^{\prime}(t), \gamma^{\prime}(t)\right) & =\operatorname{Ric}\left(e_{1}, e_{1}\right) \\
& \geq-\lambda-\frac{1}{2} \mathcal{L}_{V} g\left(e_{1}, e_{1}\right) \\
& =-\lambda-<\nabla_{e_{1}} V, e_{1}> \\
& =-\lambda-e_{1}<V, e_{1}>+<V, \nabla_{e_{1}} e_{1}> \\
& =-\lambda-\frac{\partial}{\partial t}<V, \gamma^{\prime}(t)>.
\end{aligned}
$$

This is the key simplification that allows us to proceed. Hence, (2.5) becomes

$$
\begin{aligned}
\Delta s-\frac{n-1}{s} & \leq \frac{1}{s^{2}} \int_{0}^{s}\left[t^{2} \frac{\partial}{\partial t}<V, \gamma^{\prime}(t)>+\lambda t^{2}\right] d t \\
& \leq \frac{\lambda}{3} s+\frac{1}{s^{2}}\left[t^{2}<V, \gamma^{\prime}(t)>\left.(\gamma(t))\right|_{0} ^{s}-2 \int_{0}^{s} t<V, \gamma^{\prime}(t)>d t\right] \\
& \leq \frac{\lambda}{3} s+<V, \nabla s>-\frac{2}{s^{2}} \int_{0}^{s} t<V, \gamma^{\prime}(t)>d t
\end{aligned}
$$

Denote $d_{0}=d(x, O)$.

Case 1: If $s \leq d_{0}$, then by the triangle inequality, we have

$$
d(\gamma(t), O) \geq d_{0}-t .
$$

Hence,

$$
\begin{aligned}
-\frac{2}{s^{2}} \int_{0}^{s} t<V, \gamma^{\prime}(t)>d t & \leq \frac{1}{s^{2}} \int_{0}^{s} 2 t \cdot \frac{K}{\left(d_{0}-t\right)^{\alpha}} d t \\
& \leq \frac{C(\alpha) K}{s}\left[-\left.\left(d_{0}-t\right)^{1-\alpha}\right|_{0} ^{s}\right] \\
& =\frac{C(\alpha) K}{s}\left[d_{0}^{1-\alpha}-\left(d_{0}-s\right)^{1-\alpha}\right] \\
& \leq \frac{C(\alpha) K}{s^{\alpha}}
\end{aligned}
$$

In the last step above, we have used the fact that

$$
s^{1-\alpha}+\left(d_{0}-s\right)^{1-\alpha} \geq d_{0}^{1-\alpha} .
$$

Indeed, the above inequality can be obtained by setting $x=\frac{s}{d_{0}-s}$ in the inequality

$$
x^{1-\alpha}+1 \geq(1+x)^{1-\alpha}, \quad x>0 .
$$


By plugging (2.7) into (2.6), one has

$$
\Delta s-\frac{n-1}{s} \leq \frac{\lambda}{3} s+<V, \nabla s>+\frac{C(\alpha) K}{s^{\alpha}} .
$$

Case 2: If $s>d_{0}$, then for any $d_{0} \leq t \leq s$, we have

$$
d(\gamma(t), O) \geq t-d_{0},
$$

and

$$
\begin{aligned}
-\frac{2}{s^{2}} \int_{0}^{s} t<V, \gamma^{\prime}(t)>d t & \leq \frac{1}{s^{2}} \int_{0}^{d_{0}} 2 t \cdot \frac{K}{\left(d_{0}-t\right)^{\alpha}} d t+\frac{1}{s^{2}} \int_{d_{0}}^{s} 2 t \cdot \frac{K}{\left(t-d_{0}\right)^{\alpha}} d t \\
& \leq \frac{C(\alpha) K d_{0}}{s^{2}} d_{0}^{1-\alpha}+\frac{C(\alpha) K\left(s-d_{0}\right)^{1-\alpha}}{s} \\
& =\frac{C(\alpha) K}{s^{\alpha}} .
\end{aligned}
$$

Therefore, from (2.11), we deduce

$$
\Delta s-\frac{n-1}{s} \leq \frac{\lambda}{3} s+<V, \nabla s>+\frac{C(\alpha) K}{s^{\alpha}} .
$$

This finishes the proof of the proposition.

Here is the main result of this subsection.

Theorem 2.2 (Volume comparison). Assume that (2.1) and (2.2) hold.

(a). Let $w(s, \cdot)$ denote the volume element of the metric $g$ on $\mathbf{M}$ in geodesic polar coordinates. Then for any $0<s_{1}<s_{2}$, we have

$$
\frac{w\left(s_{2}, \cdot\right)}{s_{2}^{n-1}} \leq e^{C(\alpha) K s_{2}^{1-\alpha}+\lambda s_{2}^{2}} \frac{w\left(s_{1}, \cdot\right)}{s_{1}^{n-1}} .
$$

In particular, by letting $s_{1} \rightarrow 0$, we get

$$
w(s, \cdot) \leq e^{C(\alpha) K s^{1-\alpha}+\lambda s^{2}} s^{n-1}, \quad \forall s>0,
$$

and hence

$$
\operatorname{Vol}(B(x, r)) \leq e^{C(\alpha) K r^{1-\alpha}+\lambda r^{2}} \frac{\operatorname{Vol}\left(S^{n-1}\right)}{n} r^{n}, \quad \forall r>0 .
$$

Here, $S^{n-1}$ is the unit sphere in $\mathbb{R}^{n}$.

(b). Suppose in addition that the volume non-collapsing condition holds

$$
\operatorname{Vol}(B(x, 1)) \geq \rho,
$$

for all $x \in \mathbf{M}$ and some constant $\rho>0$. Then for any $0<r_{1}<r_{2} \leq 1$, we have the volume ratio bound

$$
\frac{\operatorname{Vol}\left(B\left(x, r_{2}\right)\right)}{r_{2}^{n}} \leq e^{C(n, \lambda, K, \alpha, \rho)\left[\lambda\left(r_{2}^{2}-r_{1}^{2}\right)+K\left(r_{2}-r_{1}\right)^{1-\alpha}\right]} \cdot \frac{\operatorname{Vol}\left(B\left(x, r_{1}\right)\right)}{r_{1}^{n}} .
$$

(c). In particular, suppose that the Bakry-Émery Ricci curvature tensor Ric + Hess $L$ satisfies

and

$$
\text { Ric }+ \text { Hess } L \geq-\lambda g,
$$

$$
|\nabla L(y)| \leq \frac{K}{d(y, O)^{\alpha}}
$$


for all $y \in \mathbf{M}$, a fixed point $O \in \mathbf{M}$, and constants $\lambda \geq 0, K \geq 0, \alpha \in[0,1)$, and assume in addition that the noncollapsing condition (2.16) holds. Then the conclusions of part (a) and (b) are true.

Proof of part (a). First of all, from (2.3) we have

$$
\frac{\partial}{\partial s} \ln (w(s, \cdot))=\frac{w^{\prime}(s, \cdot)}{w(s, \cdot)}=\Delta s \leq \frac{n-1}{s}+\frac{\lambda}{3} s+<V, \nabla s>+\frac{C(\alpha) K}{s^{\alpha}} .
$$

Recall that $d_{0}=d(x, O)$.

Case 1: If $s_{1}<s_{2} \leq d_{0}$, by using the assumption (2.2) and triangle inequality, (2.20) can be transformed as

$$
\frac{\partial}{\partial s} \ln (w(s, \cdot)) \leq \frac{n-1}{s}+\frac{\lambda}{3} s+\frac{K}{\left(d_{0}-s\right)^{\alpha}}+\frac{C(\alpha) K}{s^{\alpha}} .
$$

Integrating both sides from $s_{1}$ to $s_{2}$ gives

$$
\begin{aligned}
\ln \frac{w\left(s_{2}, \cdot\right)}{w\left(s_{1}, \cdot\right)} \leq & \ln \left(\frac{s_{2}}{s_{1}}\right)^{n-1}+\frac{\lambda}{6}\left(s_{2}^{2}-s_{1}^{2}\right)+C(\alpha) K\left(s_{2}^{1-\alpha}-s_{1}^{1-\alpha}\right) \\
& +C(\alpha) K\left[\left(d_{0}-s_{1}\right)^{1-\alpha}-\left(d_{0}-s_{2}\right)^{1-\alpha}\right] \\
\leq & \ln \left(\frac{s_{2}}{s_{1}}\right)^{n-1}+\frac{\lambda}{6} s_{2}^{2}+C(\alpha) K s_{2}^{1-\alpha}+C(\alpha) K\left(s_{2}-s_{1}\right)^{1-\alpha} \\
\leq & \ln \left(\frac{s_{2}}{s_{1}}\right)^{n-1}+\frac{\lambda}{6} s_{2}^{2}+C(\alpha) K s_{2}^{1-\alpha},
\end{aligned}
$$

where in the second last step we have used (2.9) for $x=\frac{s_{2}-s_{1}}{d_{0}-s_{2}}$ to derive

$$
\left(d_{0}-s_{1}\right)^{1-\alpha}-\left(d_{0}-s_{2}\right)^{1-\alpha} \leq\left(s_{2}-s_{1}\right)^{1-\alpha} .
$$

In particular, one has for $s_{1} \leq d_{0}$,

$$
\ln \frac{w\left(d_{0}, \cdot\right)}{w\left(s_{1}, \cdot\right)} \leq \ln \left(\frac{d_{0}}{s_{1}}\right)^{n-1}+\frac{\lambda}{6} d_{0}^{2}+C(\alpha) K d_{0}^{1-\alpha} .
$$

Case 2: If $d_{0} \leq s_{1}<s_{2}$, similarly since

$$
<V, \nabla s>\leq|V|(\gamma(s)) \leq \frac{K}{\left(s-d_{0}\right)^{\alpha}}
$$

we deduce from (2.20) that

$$
\begin{aligned}
\ln \frac{w\left(s_{2}, \cdot\right)}{w\left(s_{1}, \cdot\right)} \leq & \ln \left(\frac{s_{2}}{s_{1}}\right)^{n-1}+\frac{\lambda}{6}\left(s_{2}^{2}-s_{1}^{2}\right)+C(\alpha) K\left(s_{2}^{1-\alpha}-s_{1}^{1-\alpha}\right) \\
& +C(\alpha) K\left[\left(s_{2}-d_{0}\right)^{1-\alpha}-\left(s_{1}-d_{0}\right)^{1-\alpha}\right] \\
\leq & \ln \left(\frac{s_{2}}{s_{1}}\right)^{n-1}+\frac{\lambda}{6} s_{2}^{2}+C(\alpha) K s_{2}^{1-\alpha}+C(\alpha) K\left(s_{2}-s_{1}\right)^{1-\alpha} \\
\leq & \ln \left(\frac{s_{2}}{s_{1}}\right)^{n-1}+\frac{\lambda}{6} s_{2}^{2}+C(\alpha) K s_{2}^{1-\alpha} .
\end{aligned}
$$

Especially, it implies that for $s_{2} \geq d_{0}$,

$$
\ln \frac{w\left(s_{2}, \cdot\right)}{w\left(d_{0}, \cdot\right)} \leq \ln \left(\frac{s_{2}}{d_{0}}\right)^{n-1}+\frac{\lambda}{6} s_{2}^{2}+C(\alpha) K s_{2}^{1-\alpha} .
$$


Case 3: If $s_{1} \leq d_{0} \leq s_{2}$, then by adding (2.22) to (2.24), we get

$$
\ln \frac{w\left(s_{2}, \cdot\right)}{w\left(s_{1}, \cdot\right)} \leq \ln \left(\frac{s_{2}}{s_{1}}\right)^{n-1}+\frac{\lambda}{6} s_{2}^{2}+C(\alpha) K s_{2}^{1-\alpha} .
$$

Now (2.13) is a direct consequence of (2.21), (2.23), and (2.25). This proves part (a) of the theorem.

In order to prove part (b) of the theorem, we need two more elementary results besides Proposition 2.1.

First, we present a result which is essentially known in [PeWe1. We give a proof for completeness.

Lemma 2.3. Let $B(x, r)$ denote the geodesic ball in $\mathbf{M}$ centered at $x$ with radius $r$, and $\operatorname{Vol}(B(x, r))$ denote the volume of $B(x, r)$. Then it holds that

$$
\frac{d}{d r}\left(\frac{\operatorname{Vol}(B(x, r))}{r^{n}}\right) \leq \frac{1}{r^{n+1}} \int_{S^{n-1}} \int_{0}^{r} s \psi w(s, \theta) d s d \theta,
$$

where $\psi=\left(\Delta s-\frac{n-1}{s}\right)_{+}=\max \left\{\Delta s-\frac{n-1}{s}, 0\right\}, S^{n-1}$ denotes the unit sphere in $\mathbb{R}^{n}$ and $d \theta$ is its volume element.

Proof. Notice that

$$
\frac{d}{d r} \operatorname{Vol}(B(x, r))=\frac{d}{d r} \int_{0}^{r} \int_{S^{n-1}} w(t, \theta) d \theta d t=\int_{S^{n-1}} w(r, \cdot) d \theta .
$$

Therefore,

$$
\begin{aligned}
& \frac{d}{d r}\left(\frac{\operatorname{Vol}(B(x, r))}{r^{n}}\right)=\frac{r^{n} \int_{S^{n-1}} w(r, \theta) d \theta-n r^{n-1} \operatorname{Vol}(B(x, r))}{r^{2 n}} \\
& =\frac{n \int_{S^{n-1}} w(r, \theta) d \theta \int_{0}^{r} t^{n-1} d t-n r^{n-1} \int_{0}^{r} \int_{S^{n-1}} w(t, \theta) d \theta d t}{r^{2 n}} \\
& =\frac{n}{r^{2 n}} \int_{S^{n-1}} \int_{0}^{r}\left[t^{n-1} w(r, \theta)-r^{n-1} w(t, \theta)\right] d t d \theta \\
& =\frac{n}{r^{2 n}} \int_{S^{n-1}} \int_{0}^{r} t^{n-1} r^{n-1}\left[\frac{w(r, \theta)}{r^{n-1}}-\frac{w(t, \theta)}{t^{n-1}}\right] d t d \theta \\
& =\frac{n}{r^{n+1}} \int_{S^{n-1}} \int_{0}^{r} t^{n-1}\left(\int_{t}^{r} \frac{d}{d s}\left[\frac{w(s, \theta)}{s^{n-1}}\right] d s\right) d t d \theta \\
& =\frac{n}{r^{n+1}} \int_{S^{n-1}} \int_{0}^{r} t^{n-1}\left(\int_{t}^{r}\left[\frac{s w^{\prime}(s, \cdot)-(n-1) w(s, \cdot)}{s^{n}}\right] d s\right) d t d \theta \\
& \leq \frac{n}{r^{n+1}} \int_{S^{n-1}}\left(\int_{0}^{r} \int_{t}^{r} t^{n-1} \frac{\psi w(s, \theta)}{s^{n-1}} d s d t\right) d \theta \quad\left(\text { by } w^{\prime}=w \Delta s\right) \text {, } \\
& =\frac{n}{r^{n+1}} \int_{S^{n-1}}\left(\int_{0}^{r} \int_{0}^{s} t^{n-1} \frac{\psi w(s, \theta)}{s^{n-1}} d t d s\right) d \theta \\
& =\frac{1}{r^{n+1}} \int_{S^{n-1}} \int_{0}^{r} s \psi w(s, \theta) d s d \theta \text {. }
\end{aligned}
$$


We will also need the following

Lemma 2.4. Suppose that in addition to the assumptions (2.1) and (2.2), the following volume noncollapsing condition holds

$$
\operatorname{Vol}(B(x, 1)) \geq \rho,
$$

for all $x \in \mathbf{M}$ and some constant $\rho>0$. Then for any $q \in\left(0, \frac{n}{\alpha}\right)$ we have

$$
\sup _{x \in \mathbf{M}, 0<r \leq 1} r^{\alpha}\|V\|_{q, B(x, r)}^{*} \leq C(n, K, \lambda, \alpha, \rho, q) K
$$

where

$$
\|V\|_{q, B(x, r)}^{*}=\left(\oint_{B(x, r)}|V|^{q} d g\right)^{1 / q}=\left(\frac{1}{\operatorname{Vol}(B(x, r))} \int_{B(x, r)}|V|^{q} d g\right)^{1 / q} .
$$

Proof. For any $0<r \leq 1$, according to the assumption (2.2),

$$
\|V\|_{q, B(x, r)}^{*} \leq\left(\frac{1}{\operatorname{Vol}(B(x, r))} \int_{B(x, r)} \frac{K^{q}}{d(y, O)^{\alpha q}} d g(y)\right)^{1 / q} .
$$

Case 1: If $d(x, O) \leq 2 r$, then $B(x, r) \subset B(O, 3 r)$, then for any $0<q<\frac{n}{\alpha}$, we have

$$
\int_{B(x, r)} \frac{1}{d(y, O)^{\alpha q}} d g(y) \leq \int_{B(O, 3 r)} \frac{1}{d(y, O)^{\alpha q}} d g(y) \leq C(n, \alpha, q) e^{C(\alpha) K r^{1-\alpha}+\lambda r^{2}} r^{n-\alpha q} .
$$

Indeed, by (2.14), for any $\gamma<n$, the following holds,

$$
\begin{aligned}
\int_{B(O, r)} \frac{1}{d(y, O)^{\gamma}} d g(y) & =\int_{S^{n-1}} \int_{0}^{r} \frac{1}{s^{\gamma}} w(s, \theta) d s d \theta \\
& =\int_{S^{n-1}} \sum_{k=1}^{\infty} \int_{\frac{r}{2^{k}}}^{\frac{r}{2^{k-1}}} \frac{1}{s^{\gamma}} w(s, \theta) d s d \theta \\
& \leq \int_{S^{n-1}} \sum_{k=1}^{\infty} \frac{2^{k \gamma}}{r^{\gamma}} \int_{\frac{r}{2^{k}}}^{\frac{r}{2^{k-1}}} e^{C(\alpha) K s^{1-\alpha}+\lambda s^{2} s^{n-1} d s d \theta} \\
& \leq e^{C(\alpha) K r^{1-\alpha}+\lambda r^{2}} \int_{S^{n-1}} \sum_{k=1}^{\infty} \frac{2^{k \gamma}}{r^{\gamma}} \cdot \frac{r^{n}}{2^{n(k-1)}} d \theta \\
& \leq C(n, \gamma) e^{C(\alpha) K r^{1-\alpha}+\lambda r^{2}} r^{n-\gamma} .
\end{aligned}
$$

Also, by (2.14) and the noncollapsing assumption (2.27), for $r \leq 1$ one has

$$
\begin{aligned}
\operatorname{Vol}(B(x, r)) & =\int_{S^{n-1}} \int_{0}^{r} w(s, \theta) d s d \theta \\
& =r \int_{S^{n-1}} \int_{0}^{1} w(r t, \theta) d t d \theta \\
& \geq r \int_{S^{n-1}} \int_{0}^{1} \frac{1}{e^{C(\alpha) K+\lambda}} r^{n-1} w(t, \theta) d t d \theta \\
& =\frac{\rho}{e^{C(\alpha) K+\lambda}} r^{n}
\end{aligned}
$$


This and (2.30) imply that

$$
\frac{1}{\operatorname{Vol}(B(x, r))} \int_{B(O, r)} \frac{1}{d(y, O)^{\alpha q}} d g(y) \leq C(n, \alpha, q) e^{C(\alpha) K r^{1-\alpha}+\lambda r^{2}} \frac{e^{C(\alpha) K+\lambda}}{\rho} r^{-\alpha q} .
$$

Case 2: If $d(x, O)>2 r$, then $d(y, O) \geq d(x, O)-d(x, y) \geq r$ for all $y \in B(x, r)$. Thus,

$$
\int_{B(x, r)} \frac{1}{d(y, O)^{\alpha q}} d g(y) \leq r^{-\alpha q} \operatorname{Vol}(B(x, r)) .
$$

So in both cases we obtain

$$
\|V\|_{q, B(x, r)}^{*} \leq C(n, \lambda, K, \alpha, \rho, q) K r^{-\alpha} .
$$

This finishes the proof of the lemma.

Now we are ready to prove part (b) of Theorem 2.2

Proof of part (b). Recall that $\psi=\left(\Delta s-\frac{n-1}{s}\right)_{+}$. From (2.3), we have

$$
\psi \leq \frac{\lambda}{3} s+\frac{C(\alpha) K}{s^{\alpha}}+|V|
$$

Notice that by (2.28), we have

$$
\oint_{B(x, r)}|V| d g \leq \frac{C(n, \lambda, K, \alpha, \rho) K}{r^{\alpha}} .
$$

Let $Q(r)=\frac{\operatorname{Vol}(B(x, r))}{r^{n}}$, then (2.26)

$$
\begin{aligned}
\frac{d}{d r} Q(r) & \leq \frac{1}{r^{n+1}} \int_{S^{n-1}} \int_{0}^{r} s\left(\frac{\lambda}{3} s+\frac{C(\alpha) K}{s^{\alpha}}+|V|\right) w(s, \theta) d s d \theta \\
& \leq\left[\frac{\lambda r}{3}+\frac{C(n, \lambda, K, \alpha, \rho) K}{r^{\alpha}}\right] Q(r) .
\end{aligned}
$$

The proof of the theorem is finished after integrating both sides of the above inequality.

Finally, the proof of part (c) follows directly by considering the special case where $V=\nabla L$ in (2.1).

In the following, we show that under a stronger assumption that $|V|$ is bounded, the results above can be improved. Especially, we can obtain the volume comparison theorem without assuming the volume noncollapsing condition (2.16).

Corollary 2.5. Suppose that (2.1) holds and moreover

$$
|V| \leq K
$$

Then the following conclusions are true.

(a). Let $s=d(y, x)$ be the distance from any point $y$ to some fixed point $x$, and $\gamma:[0, s] \rightarrow \mathbf{M}$ a normal minimal geodesic with $\gamma(0)=x$ and $\gamma(s)=y$. Then in the distribution sense,

$$
\Delta s-\frac{n-1}{s} \leq \frac{\lambda}{3} s+2 K
$$


(b). Let $w(s, \cdot)$ denote the volume element of the metric $g$ on $\mathbf{M}$ under geodesic polar coordinates. Then for any $0<s_{1}<s_{2}$, we have

$$
\frac{w\left(s_{2}, \cdot\right)}{s_{2}^{n-1}} \leq e^{2 K s_{2}+\lambda s_{2}^{2}} \frac{w\left(s_{1}, \cdot\right)}{s_{1}^{n-1}} .
$$

In particular, by letting $s_{1} \rightarrow 0$, we get

$$
w(s, \cdot) \leq e^{2 K s+\lambda s^{2}} s^{n-1}, \quad \forall s>0
$$

and hence

$$
\operatorname{Vol}(B(x, r)) \leq e^{2 K r+\lambda r^{2}} \frac{\operatorname{Vol}\left(S^{n-1}\right)}{n} r^{n}, \quad \forall r>0 .
$$

(c). For any $0<r_{1}<r_{2}$, we have

$$
\frac{\operatorname{Vol}\left(B\left(x, r_{2}\right)\right)}{r_{2}^{n}} \leq e^{\left[\lambda\left(r_{2}^{2}-r_{1}^{2}\right)+2 K\left(r_{2}-r_{1}\right)\right]} \cdot \frac{\operatorname{Vol}\left(B\left(x, r_{1}\right)\right)}{r_{1}^{n}} .
$$

Proof. When $|V|$ is bounded, Lemma 2.4 holds without any other condition for $\alpha=0$ and $q=\infty$. The proof of the corollary is just special cases of those of Proposition 2.1, Theorem 2.2 with the parameter $\alpha=0$.

Remark 2.6. Following the proofs above verbatim, one can see that similar results also hold if we relax assumption (2.1) to

$$
R i c+\frac{1}{2} \mathcal{L}_{V} g-\frac{1}{N-n} V \otimes V \geq-\lambda g,
$$

and require the constant $\alpha$ in assumption (2.2) to be less than $1 / 2$. Here $N>n$ is any constant, and when $N=\infty$, we arrive at (2.1).

2.2. Bakry-Émery Ricci condition. Next we consider the special case when $V$ is a gradient field, namely we are returning to the Bakry-Émery Ricci curvature. Let $V=\nabla L$ for some smooth function $L$ and assume

$$
\operatorname{Ric}+\operatorname{Hess} L \geq-\lambda g
$$

for some constant $\lambda \geq 0$. Since one can perform integration by parts twice, the condition on the gradient of the potential can be relaxed to an integral one.

In this case, we will impose the following conditions on $L$ :

$$
|L(y)-L(z)| \leq K_{1} d(y, z)^{\alpha} \text {, and } \sup _{x \in \mathbf{M}, 0 \leq r \leq 1}\left(r^{\beta}\|\nabla L\|_{q, B(x, r)}^{*}\right) \leq K_{2}
$$

for any $y, z \in \mathbf{M}$ with $d(y, z) \leq 1$. Here $K_{1}, K_{2} \geq 0,0<\alpha<1,0 \leq \beta<1$, and $q \geq 1$ are constants, and

$$
\|\nabla L\|_{q, B(x, r)}^{*}=\left(\oint_{B(x, r)}|\nabla L|^{q}(y) d V(y)\right)^{1 / q} .
$$

Comparing with the more general case earlier, we no longer need to assume $\mathbf{M}$ being non-collapsed. Intuitively, the above assumptions on the potential function $L$ is that it is Hölder continuous and $|\nabla L|$ has singularity of order less 1. These include not only the popular special case when $|\nabla L|$ is bounded but also Ricci solitons with just bounded Ricci potentials. More detailed discussion will be given later in this section. Since we are mainly concerned with local properties, the assumption on $L$ is restricted to balls of size 1 for convenience. The condition on $\nabla L$ is almost sharp by scaling considerations. 
Theorem 2.7. Suppose that (2.43) and (2.44) are satisfied.

(a) Let $s=d(x, y)$ be the distance from any point $y$ to some fixed point $x$, and $\gamma$ : $[0, s] \rightarrow \mathbf{M}$ a normal minimal geodesic with $\gamma(0)=x$ and $\gamma(s)=y$. Then the following inequalities hold in the sense of distribution.

$$
\Delta s-\frac{n-1}{s} \leq \frac{\lambda}{3} s+\frac{4 K_{1}}{s^{1-\alpha}}+<\nabla L, \nabla s>, \quad \forall s<1
$$

moreover,

$$
\frac{\partial}{\partial s} \frac{w(s, \theta)}{s^{n-1}} \leq\left[\frac{\lambda}{3} s+\frac{4 K_{1}}{s^{1-\alpha}}+<\nabla L, \nabla s>\right] \frac{w(s, \theta)}{s^{n-1}} .
$$

Here $w=w(s, \theta)$ is the volume element in the geodesic polar coordinates which is regarded as 0 on the cut locus.

(b) For any $0<r_{1}<r_{2} \leq 1$, we have

$$
\frac{\operatorname{Vol}\left(B\left(x, r_{2}\right)\right)}{r_{2}^{n}} \leq e^{\lambda\left(r_{2}^{2}-r_{1}^{2}\right)+K_{2}\left(r_{2}-r_{1}\right)^{1-\beta}+4 K_{1}\left(r_{2}-r_{1}\right)^{\alpha}} \cdot \frac{\operatorname{Vol}\left(B\left(x, r_{1}\right)\right)}{r_{1}^{n}} .
$$

Proof of (a). Again, we just prove the inequalities at smooth points of $s$.

First of all, from (2.5), we have

$$
\Delta s \leq \frac{n-1}{s}-\frac{1}{s^{2}} \int_{0}^{s} t^{2} \operatorname{Ric}\left(\frac{\partial}{\partial t}, \frac{\partial}{\partial t}\right) d s .
$$

According to (2.43), along the geodesic $\gamma(t)$, one has

$$
\operatorname{Ric}\left(\partial_{t}, \partial_{t}\right) \geq-\lambda-\operatorname{Hess} L\left(\partial_{t}, \partial_{t}\right)=-\lambda-\frac{d^{2}}{d t^{2}} L(\gamma(t))
$$

By using (2.49) and integration by parts, we can rewrite (2.48) as

$$
\begin{aligned}
\Delta s-\frac{n-1}{s} & \leq \frac{1}{s^{2}} \int_{0}^{s}\left[t^{2} \frac{d^{2}}{d t^{2}} L(\gamma(t))+\lambda t^{2}\right] d t \\
& \leq \frac{\lambda}{3} s+\frac{1}{s^{2}}\left[\left.t^{2} \frac{d}{d t} L(\gamma(t))\right|_{0} ^{s}-2 \int_{0}^{s} t \frac{d}{d t} L(\gamma(t)) d t\right] \\
& \leq \frac{\lambda}{3} s+<\nabla L, \nabla s>-\frac{2}{s^{2}} \int_{0}^{s} t \frac{d}{d t} L(\gamma(t)) d t \\
& =\frac{\lambda}{3} s+<\nabla L, \nabla s>-\frac{2}{s} L(\gamma(s))+\frac{2}{s^{2}} \int_{0}^{s} L(\gamma(t)) d t \\
& =\frac{\lambda}{3} s+<\nabla L, \nabla s>-\frac{2}{s}[L(\gamma(s))-L(\gamma(0))]+\frac{2}{s^{2}} \int_{0}^{s}[L(\gamma(t))-L(\gamma(0))] d t \\
& \leq \frac{\lambda}{3} s+<\nabla L, \nabla s>+\frac{4 K_{1}}{s^{1-\alpha}} .
\end{aligned}
$$

This proves (2.45). From this, (2.46) follows by the formula $\partial_{s} w=\Delta s w$.

Proof of (b). From (2.45), we have

$$
\psi \leq \frac{\lambda}{3} s+\frac{4 K_{1}}{s^{1-\alpha}}+|\nabla L|
$$


Notice that by (2.44), we have

$$
\oint_{B(x, r)}|\nabla L| d V \leq\left(\oint_{B(x, r)}|\nabla L|^{q} d V\right)^{1 / q} \leq \frac{K_{2}}{r^{\beta}} .
$$

Let $Q(r)=\frac{\operatorname{Vol}(B(x, r))}{r^{n}}$, then $(2.51)$ and Proposition 2.3 imply that

$$
\begin{aligned}
\frac{d}{d r} Q(r) & \leq \frac{1}{r^{n+1}} \int_{\mathbb{S}^{n-1}} \int_{0}^{r} s\left(\frac{\lambda}{3} s+\frac{4 K_{1}}{s^{1-\alpha}}+|\nabla L|\right) w(s, \theta) d s d \theta \\
& \leq\left(\frac{\lambda r}{3}+\frac{4 K_{1}}{r^{1-\alpha}}+\frac{K_{2}}{r^{\beta}}\right) Q(r) .
\end{aligned}
$$

This finishes the proof of part (b) after integrating both sides of the above inequality.

It is worth mentioning that, when $V=\nabla L$ in (2.1), the assumption (2.44) is actually a weaker condition than (2.19) and (2.16) due to the following lemma.

Lemma 2.8. Suppose that

$$
\text { Ric }+\operatorname{Hess} L \geq-\lambda g,
$$

and assume that (2.19) and (2.16) hold, i.e.,

$$
|\nabla L(x)| \leq \frac{K}{d(x, O)^{\alpha}}, \quad \text { and } \quad \operatorname{Vol}(B(x, 1)) \geq \rho .
$$

Then for any $q \in\left(n, \frac{n}{\alpha}\right)$, we have

$$
|L(y)-L(z)| \leq \frac{2 K}{1-\alpha} d(y, z)^{1-\alpha}
$$

for any $y, z \in \mathbf{M}$, and

$$
\sup _{x \in \mathbf{M}, 0<r \leq 1} r^{\alpha}\|\nabla L\|_{q, B(x, r)}^{*} \leq C(n, K, \lambda, \alpha, \rho) K .
$$

Proof. Since the proof of (2.53) is given in Lemma 2.4, we only need to show (2.52). Suppose that $\gamma:[0, d(y, z)] \rightarrow \mathbf{M}$ is a minimal geodesic from $y$ to $z$. We separate the proof into two cases.

Case 1: If $d(y, O) \geq d(y, z)$, then for any $t \in[0, d(y, z)], d(\gamma(t), O) \geq d(y, O)-d(y, \gamma(t))=$ $d(y, O)-t$. Thus,

$$
\begin{aligned}
|L(y)-L(z)| & =\left|\int_{0}^{d(y, z)}<\nabla L, \gamma^{\prime}(t)>d t\right| \\
& \leq K \int_{0}^{d(y, z)} \frac{1}{[d(y, O)-t]^{\alpha}} d t \\
& =\frac{K}{1-\alpha}\left(d(y, O)^{1-\alpha}-[d(y, O)-d(y, z)]^{1-\alpha}\right) \\
& \leq \frac{K}{1-\alpha} d(y, z)^{1-\alpha} .
\end{aligned}
$$


Case 2: If $d(y, O)<d(y, z)$, then

$$
\begin{aligned}
|L(y)-L(z)| & \leq K \int_{0}^{d(y, z)} \frac{1}{d(\gamma(t), O)^{\alpha}} d t \\
& =K \int_{0}^{d(y, O)} \frac{1}{d(\gamma(t), O)^{\alpha}} d t+K \int_{d(y, O)}^{d(y, z)} \frac{1}{d(\gamma(t), O)^{\alpha}} d t \\
& \leq K \int_{0}^{d(y, O)} \frac{1}{[d(y, O)-t]^{\alpha}} d t+K \int_{d(y, O)}^{d(y, z)} \frac{1}{[t-d(y, O)]^{\alpha}} d t \\
& =\frac{K}{1-\alpha}\left(d(y, O)^{1-\alpha}+[d(y, z)-d(y, O)]^{1-\alpha}\right) \\
& \leq \frac{2 K}{1-\alpha} d(y, z)^{1-\alpha} .
\end{aligned}
$$

This proves (2.52), and hence finishes the proof of the lemma.

The next corollary treats the case of gradient Ricci solitons for which the results are the strongest. A complete Riemannian manifold $\left(M, g_{i j}\right)$ is a so called gradient Ricci soliton if the following equation is satisfied

$$
R_{i j}+\nabla_{i} \nabla_{j} L=\lambda g_{i j}
$$

where $\lambda$ is a constant, and $L$ is a smooth function on $\mathbf{M}$. The cases where $\lambda>0,=0$, and $<0$ correspond to shrinking, steady and expanding solitons, respectively.

Ricci solitons not only represent self-similar solutions of the Ricci flow, but more importantly, they appear as singularity models, i.e., the dilation limits of singular solutions, in many circumstances in the Ricci flow. Thus, the study of Ricci solitons is critical for the singularity analysis in the Ricci flow. We refer readers to Cao1, Cao2 and references therein for more information on the Ricci solitons.

For a Ricci soliton (2.54), it is well known that

$$
R+|\nabla L|^{2}-2 \lambda L=C_{0},
$$

where $R$ is the scalar curvature of $\mathbf{M}$, and $C_{0}$ is a constant. Therefore, for shrinking and expanding solitons, i.e., when $\lambda \neq 0$, we may add $L$ by constant so that it satisfies the normalization condition

$$
R+|\nabla L|^{2}-2 \lambda L=0 .
$$

For steady soliton, the above equation becomes

$$
R+|\nabla L|^{2}=C_{0}
$$

If $C_{0}=0$, then nothing needs to be done. Actually, Z.-H. Zhang [ZZ] and B.-L. Chen Chen showed that $R \geq 0$ for steady solitons. Thus, when $C_{0}=0$, one immediately gets from (2.57) that $R \equiv 0$. Then by the following evolution equation of $R$,

$$
\Delta R=<\nabla R, \nabla L>+2|R i c|^{2},
$$

we obtain Ric $\equiv 0$.

Therefore, for steady solitons, let us just consider the case where $C_{0} \neq 0$ in (2.57). However, in this case, it is impossible to normalize the soliton so that $C_{0}=0$. On the 
other hand, we may rescale the metric $g$ by a constant, so that the steady soliton can be normalized as

$$
R+|\nabla L|^{2}=1
$$

Assume that $(M, g)$ is a gradient Ricci soliton with normalization (2.56) if it is a shrinking or expanding soliton, or (2.58) if it is a steady soliton. Again, by the result in [ZZh] and Chen, we have $R \geq 0$ when $\lambda \geq 0$, and $R \geq-C_{1}(n)$ when $\lambda<0$. Hence, if we further assume $|L| \leq K$, then it follows from (2.56) and (2.58) that

$$
|\nabla L| \leq \Lambda(n, \lambda, K)
$$

where

$$
\Lambda(n, \lambda, K)= \begin{cases}\sqrt{2 \lambda K}, & \text { if } \lambda>0 \\ 1, & \text { if } \lambda=0 \\ \sqrt{-2 \lambda K+C_{1}(n)}, & \text { if } \lambda<0\end{cases}
$$

This shows that the assumptions in Corollary 2.5 are satisfied. Therefore, based on the previous argument and Corollary 2.5, we have

Corollary 2.9. (Gradient Ricci soliton case) Suppose that $\left(M, g_{i j}\right)$ is a gradient Ricci soliton (2.54) satisfying (2.56) when $\lambda \neq 0$ or (2.58) when $\lambda=0$. Assume further that

$$
|L| \leq K, \text { in } B(O, 2 \delta)
$$

for some fixed point $O \in \mathbf{M}$ and radius $\delta$. Let $\Lambda(n, \lambda, K)$ be the constant in (2.60). Then the following statements hold.

(a). Let $s=d(y, x)$ be the distance between any two points $x, y \in B(O, \delta)$, and $\gamma:[0, s] \rightarrow \mathbf{M}$ a normal minimal geodesic with $\gamma(0)=x$ and $\gamma(s)=y$. Then in the distribution sense,

$$
\Delta s-\frac{n-1}{s} \leq \frac{|\lambda|}{3} s+2 \Lambda(n, \lambda, K) .
$$

(b). Let $s$ be defined as in (a), and $w(s, \cdot)$ denote the volume element of the metric $g$ under geodesic polar coordinates. Then for any $0<s_{1}<s_{2}<d(x, \partial B(O, \delta))$, we have

$$
\frac{w\left(s_{2}, \cdot\right)}{s_{2}^{n-1}} \leq e^{2 \Lambda(n, \lambda, K) s_{2}+|\lambda| s_{2}^{2}} \frac{w\left(s_{1}, \cdot\right)}{s_{1}^{n-1}} .
$$

In particular, by letting $s_{1} \rightarrow 0$, we get

$$
w(s, \cdot) \leq e^{2 \Lambda(n, \lambda, K) s+|\lambda| s^{2}} s^{n-1},
$$

for any $0<s<d(x, \partial B(O, \delta))$, and hence

$$
\operatorname{Vol}(B(x, r)) \leq C(n) e^{2 \Lambda(n, \lambda, K) r+|\lambda| r^{2}} r^{n},
$$

for any $0<r<d(x, \partial B(O, \delta))$.

(c). For any $x \in B(O, \delta)$ and $0<r_{1}<r_{2}<d(x, \partial B(O, \delta))$, we have

$$
\frac{\operatorname{Vol}\left(B\left(x, r_{2}\right)\right)}{r_{2}^{n}} \leq e^{\left[|\lambda|\left(r_{2}^{2}-r_{1}^{2}\right)+2 \Lambda(n, \lambda, K)\left(r_{2}-r_{1}\right)\right]} \cdot \frac{\operatorname{Vol}\left(B\left(x, r_{1}\right)\right)}{r_{1}^{n}} .
$$

Remark 2.10. Note that this is a local volume ratio comparison result for gradient Ricci solitons. A volume upper bound in large scale for gradient shrinking solitons is proven in H.D. Cao and D.T. Zhou [CZ]. 
Remark 2.11. For gradient shrinking Ricci solitons, Z.L. Zhang [zlZh] showed that if the volume of $M$ is bounded below and the diameter is bounded above, then the potential function is bounded, and after a conformal transformation, the Ricci curvature also becomes bounded.

\section{LOCAL ISOPERIMETRIC AND SOBOLEV CONSTANTS ESTIMATES}

In this section, we use the volume comparison result in the previous section and follow the technique and arguments in X.Z. Dai-G.F. Wei-Z.L. Zhang [DWZ] to prove isoperimetric inequality and Sobolev inequality on manifolds satisfying the modified Ricci bound. The basic assumptions are (2.1), (2.2) and the volume noncollapsing condition (2.16). However if assumption (2.1), (2.2) hold with $\alpha=0$, namely the vector field $V$ is bounded, then the noncollapsing condition is not necessary.

Proposition 3.1. Assume that the basic assumptions (2.1), (2.2) and the noncollapsing condition (2.16) hold. Or just assume that (2.1), (2.2) hold for $\alpha=0$. There exists $r_{0}=r_{0}(n, \lambda, K, \alpha, \rho)$ and $\delta=\delta(n)$, such that for any $r \leq r_{0}$,

$$
\frac{\operatorname{Vol}(B(x, \delta r))}{\operatorname{Vol}(B(x, r))} \leq \frac{1}{2}
$$

Proof. First of all, if follows from Theorem 2.2 (b) that, for $r_{0}=r_{0}(n, \lambda, K, \alpha, \rho)<1$ such that

one has

$$
e^{C(n, \lambda, K, \alpha, \rho)\left(\lambda r_{0}^{2}+K r_{0}^{1-\alpha}\right)} \leq \frac{3}{2}
$$

$$
\frac{\operatorname{Vol}\left(B\left(x, r_{1}\right)\right)}{\operatorname{Vol}\left(B\left(x, r_{2}\right)\right)} \geq \frac{2}{3} \frac{r_{1}^{n}}{r_{2}^{n}}
$$

for any $x \in \mathbf{M}$ and $0<r_{1}<r_{2} \leq r_{0}$.

For any $r \leq r_{0}$, let $\delta<\frac{1}{3}$, and choose $x^{\prime} \in B(x, r)$ with $d=d\left(x, x^{\prime}\right)=\frac{1-\delta}{2} r \geq \frac{1}{3} r$. Then

$$
B(x, \delta r) \subset B\left(x^{\prime}, d+\delta r\right) \backslash B\left(x^{\prime}, d-\delta r\right) \subset B\left(x^{\prime}, d+\delta r\right) \subset B(x, r) .
$$

Therefore,

$$
\frac{\operatorname{Vol}(B(x, \delta r))}{\operatorname{Vol}(B(x, r))} \leq \frac{\operatorname{Vol}\left(B\left(x^{\prime}, d+\delta r\right)\right)-\operatorname{Vol}\left(B\left(x^{\prime}, d-\delta r\right)\right)}{\operatorname{Vol}\left(B\left(x^{\prime}, d+\delta r\right)\right)}=1-\frac{\operatorname{Vol}\left(B\left(x^{\prime}, d-\delta r\right)\right)}{\operatorname{Vol}\left(B\left(x^{\prime}, d+\delta r\right)\right)} .
$$

By (3.2), we have

$$
\frac{\operatorname{Vol}\left(B\left(x^{\prime}, d-\delta r\right)\right)}{\operatorname{Vol}\left(B\left(x^{\prime}, d+\delta r\right)\right)} \geq \frac{2}{3}\left(\frac{d-\delta r}{d+\delta r}\right)^{n}=\frac{2}{3}\left(\frac{1-3 \delta}{1+\delta}\right)^{n} .
$$

If we choose $\delta=\delta(n)$ small so that

$$
\left(\frac{1-3 \delta}{1+\delta}\right)^{n} \geq \frac{3}{4}
$$

then

and hence

$$
\frac{\operatorname{Vol}\left(B\left(x^{\prime}, d-\delta r\right)\right)}{\operatorname{Vol}\left(B\left(x^{\prime}, d+\delta r\right)\right)} \geq \frac{1}{2},
$$

$$
\frac{\operatorname{Vol}(B(x, \delta r))}{\operatorname{Vol}(B(x, r))} \leq \frac{1}{2} .
$$


Remark 3.2. If one assumes the volume noncollapsing condition, then we can also choose $\delta=\delta(n, \lambda, K, \alpha, \rho)$ small, such that the same conclusion holds for any $r \leq 1$.

Lemma 3.3 (Gromov Gro]). Let $\mathbf{M}^{n}$ be a complete Riemannian manifold and $H$ any hypersurface dividing $\mathbf{M}$ into two parts, $\mathbf{M}_{1}$ and $\mathbf{M}_{2}$. For any Borel subsets $W_{i} \subset \mathbf{M}_{i}$, there exists $x_{1}$ in one of $W_{i}$, say $W_{1}$, and a subset $W$ in the other part $W_{2}$, such that

$$
\operatorname{Vol}(W) \geq \frac{1}{2} \operatorname{Vol}\left(W_{2}\right)
$$

and for any $x_{2} \in W$, there is a unique minimal geodesic between $x_{1}$ and $x_{2}$ which intersects $H$ at some $z$ with

$$
d\left(x_{1}, z\right) \geq d\left(x_{2}, z\right)
$$

Lemma 3.4. Let $H, W$ and $x_{1}$ be as in the above lemma. Assume that $D=\sup _{x \in W} d\left(x_{1}, x\right) \leq$ 1 , then

$$
\operatorname{Vol}(W) \leq 2^{n-1} D \operatorname{Vol}\left(H^{\prime}\right)+\left[2^{n-2} \lambda D^{2}+C(n, \lambda, K, \alpha, \rho) D^{1-\alpha}\right] \operatorname{Vol}(B(x, D)),
$$

where $H^{\prime}$ is the set of intersection points with $H$ of geodesic $\gamma_{x_{1}, x}$ for all $x \in W$.

Proof. Let $\Gamma \subset S_{x}$ be the set of unit vectors at $x$ such that $\gamma_{v}=\gamma_{x_{1}, x_{2}}$ for some $x_{2} \in W$. Since

$$
\frac{\partial}{\partial s} \frac{w(s, \theta)}{s^{n-1}}=\frac{s \partial_{s} w-(n-1) w}{s^{n}} \leq \frac{\psi w(s, \theta)}{s^{n-1}}
$$

after integration we have

$$
w(r, \theta) \leq 2^{n-1} w(t, \theta)+2^{n-1} \int_{t}^{r} \psi(s, \theta) w(s, \theta) d s,
$$

whenever $\frac{r}{2} \leq t \leq r$.

For any $\theta \in \Gamma$, denote by $r_{1}(\theta)$ and $r_{2}(\theta)$ the minimum and maximum radius such that $\exp _{x_{1}} r \theta \in W$, respectively. Let $r(\theta)$ be the radius such that $\exp _{x_{1}}(r \theta) \in H$. Then from Lemma 3.3, we have $r(\theta) \geq \frac{1}{2} r_{2}(\theta)$, and hence

$$
\begin{aligned}
\operatorname{Vol}(W) \leq & \int_{\Gamma} \int_{r_{1}(\theta)}^{r_{2}(\theta)} w(t, \theta) d t d \theta \\
\leq & 2^{n-1} \int_{\Gamma} \int_{r_{1}(\theta)}^{r_{2}(\theta)} w(r(\theta), \theta) d t d \theta+2^{n-1} \int_{\Gamma} \int_{r_{1}(\theta)}^{r_{2}(\theta)} \int_{r(\theta)}^{t} \psi(s, \theta) w(s, \theta) d s d t d \theta \\
\leq & 2^{n-1} D \int_{\Gamma} w(r(\theta), \theta) d \theta+2^{n-1} r_{2}(\theta) \int_{\Gamma} \int_{r(\theta)}^{r_{2}(\theta)}\left[\frac{\lambda}{3} s+\frac{C(\alpha) K}{s^{\alpha}}+|V|(\gamma(s))\right] w(s, \theta) d s d \theta \\
\leq & 2^{n-1} D \operatorname{Vol}\left(H^{\prime}\right)+\left[\frac{2^{n-1}}{3} \lambda r_{2}^{2}(\theta)+C(n, \alpha) K r_{2}^{1-\alpha}(\theta)\right] \int_{\Gamma} \int_{0}^{D} w(s, \theta) d s d \theta \\
& +2^{n-1} D \int_{B(x, D)}|V| d V \\
\leq & 2^{n-1} D \operatorname{Vol}\left(H^{\prime}\right)+\left[2^{n-2} \lambda D^{2}+C(n, \lambda, K, \alpha, \rho) K D^{1-\alpha}\right] \operatorname{Vol}(B(x, D)) .
\end{aligned}
$$

In the last step above, we have used (2.36). We have also used Proposition 2.1 when going from the 2 nd to the $3 r d$ line. 
From Lemmas 3.3 and 3.4 , we immediately have

Corollary 3.5. Let $H$ be any hypersurface dividing $\mathbf{M}$ into two parts $\mathbf{M}_{1}$ and $\mathbf{M}_{2}$. For any ball $B=B(x, r), r \leq 1 / 2$, we have

$$
\begin{aligned}
& \min \left(\operatorname{Vol}\left(B \cap \mathbf{M}_{1}\right), \operatorname{Vol}\left(B \cap \mathbf{M}_{2}\right)\right) \\
\leq & 2^{n+1} r \operatorname{Vol}\left(H^{\prime}\right)+\left[2^{n} \lambda r^{2}+C(n, \lambda, K, \alpha, \rho) K r^{1-\alpha}\right] \operatorname{Vol}(B(x, 2 r)) .
\end{aligned}
$$

Corollary 3.6. Assume that (2.1), (2.2) and (2.16) hold. Or just assume that (2.1), (2.2) hold for $\alpha=0$. There is a $r_{0}=r_{0}(n, \lambda, K, \alpha, \rho)$ such that for any $r \leq r_{0}$, and geodesic ball $B(x, r)$ which is divided equally by $H$, we have

$$
\operatorname{Vol}(B(x, r)) \leq 2^{n+3} r \operatorname{Vol}(H \cap B(x, 2 r)) .
$$

Proof. By (2.17), we have

$$
\operatorname{Vol}(B(x, 2 r)) \leq 2^{n} e^{C(n, \lambda, K, \alpha, \rho)\left(\lambda r^{2}+K r^{1-\alpha}\right)} \operatorname{Vol}(B(x, r)) .
$$

Thus, to get the result in the corollary, one only needs to choose $r_{0} \leq \frac{1}{2}$ so that

$$
2^{n} e^{C(n, \lambda, K, \alpha, \rho)\left(\lambda r_{0}^{2}+K r_{0}^{1-\alpha}\right)}\left[2^{n} \lambda r_{0}^{2}+C(n, \lambda, K, \alpha, \rho) K r_{0}^{1-\alpha}\right] \leq 1 / 4 .
$$

From Proposition 3.1, Corollary 3.6 and the results in section 2, we can prove the following isoperimetric inequality.

Theorem 3.7. Assume that (2.1), (2.2) and (2.16) hold. Or just assume that (2.1), (2.2) hold for $\alpha=0$. There exists an $r_{0}=r_{0}(n, \lambda, K, \alpha, \rho)$ such that for any $r \leq r_{0}$,

$$
I D_{n}^{*}(B(x, r)) \leq C(n) r .
$$

Here $I D_{n}^{*}(B(x, r))$ is the isoperimetric constant defined by

$$
I D_{n}^{*}(B(x, r))=\operatorname{Vol}(B(x, r))^{\frac{1}{n}} \cdot \sup _{\Omega}\left\{\frac{\operatorname{Vol}(\Omega)^{\frac{n-1}{n}}}{\operatorname{Vol}(\partial \Omega)}\right\},
$$

where the supremum is taken over all smooth domains $\Omega \subset B(x, r)$ with $\partial \Omega \cap \partial B(x, r)=\emptyset$.

Proof. By Proposition 3.1, there exists an $r_{1}$ and $\delta=\delta(n)$ so that for any $r \leq r_{1}$,

$$
\frac{\operatorname{Vol}(B(x, 2 \delta r))}{\operatorname{Vol}\left(B\left(x, \frac{1}{10} r\right)\right)} \leq \frac{1}{2}, \quad \forall x \in \mathbf{M} .
$$

We will prove the theorem for any $r \leq r_{0}=\delta r_{1}$. Given any $x \in \mathbf{M}$, let $\Omega$ be a smooth connected subdomain of $B(x, r)$ whose boundary $\partial \Omega$ divides $\mathbf{M}$ into two parts $\Omega$ and $\Omega^{c}$. For any $y \in \Omega$, let $r_{y}$ be the smallest radius such that

$$
\operatorname{Vol}\left(B\left(y, r_{y}\right) \cap \Omega\right)=\operatorname{Vol}\left(B\left(y, r_{y}\right) \cap \Omega^{c}\right)=\frac{1}{2} \operatorname{Vol}\left(B\left(y, r_{y}\right)\right) .
$$

Since $\Omega \subset B(y, 2 r)$,

$$
\begin{aligned}
\operatorname{Vol}\left(B\left(y, \frac{1}{10} r\right)\right) & \geq 2 \operatorname{Vol}(B(y, 2 r)) \\
& \geq 2 \operatorname{Vol}\left(B\left(y, r_{y}\right) \cap B(y, 2 r)\right) \geq 2 \operatorname{Vol}\left(B\left(y, r_{y}\right) \cap \Omega\right)=\operatorname{Vol}\left(B\left(y, r_{y}\right)\right) .
\end{aligned}
$$

Thus, $r_{y} \leq \frac{1}{10} r$. 
By (3.5),

$$
\operatorname{Vol}\left(B\left(y, r_{y}\right)\right) \leq 2^{n+3} r_{y} \operatorname{Vol}\left(H \cap B\left(y, 2 r_{y}\right)\right)
$$

Since

$$
\Omega \subset \bigcup_{y \in \Omega} B\left(y, 2 r_{y}\right)
$$

by Vitali Covering Lemma, we may choose a countable family of disjoint balls $B_{i}=$ $B\left(y_{i}, 2 r_{y_{i}}\right)$ such that $\Omega \subset \cup_{i} B\left(y_{i}, 10 r_{y_{i}}\right)$. By (3.2), we have

$$
\frac{\operatorname{Vol}\left(B\left(y, 2 r_{y}\right)\right)}{\operatorname{Vol}\left(B\left(y, 10 r_{y}\right)\right)} \geq \frac{2}{3 \cdot 5^{n}}
$$

Hence,

$$
\sum_{i} \operatorname{Vol}\left(B_{i}\right) \geq \frac{2}{3 \cdot 5^{n}} \sum_{i} \operatorname{Vol}\left(B\left(y_{i}, 10 r_{y_{i}}\right)\right) \geq \frac{2}{3 \cdot 5^{n}} \operatorname{Vol}(\Omega),
$$

and

$$
\sum_{i} \operatorname{Vol}\left(B\left(y_{i}, r_{y_{i}}\right)\right) \geq \frac{4}{9 \cdot 10^{n}} \operatorname{Vol}(\Omega)
$$

Since $B_{i}$ 's are disjoint, from (3.5), we get

$$
\operatorname{Vol}(\partial \Omega) \geq \sum_{i} \operatorname{Vol}\left(B_{i} \cap \partial \Omega\right) \geq 2^{-(n+3)} \sum_{i} r_{y_{i}}^{-1} \operatorname{Vol}\left(B\left(y_{i}, r_{y_{i}}\right)\right)
$$

Therefore,

$$
\begin{aligned}
\frac{\operatorname{Vol}(\Omega)^{\frac{n-1}{n}}}{\operatorname{Vol}(\partial \Omega)} & \leq\left(\frac{9 \cdot 10^{n}}{4}\right)^{\frac{n-1}{n}} 2^{n+3} \frac{\left(\sum_{i} \operatorname{Vol}\left(B\left(y_{i}, r_{y_{i}}\right)\right)\right)^{\frac{n-1}{n}}}{\sum_{i} r_{y_{i}}^{-1} \operatorname{Vol}\left(B\left(y_{i}, r_{y_{i}}\right)\right)} \\
& \leq 10^{2 n+5} \frac{\sum_{i} \operatorname{Vol}\left(B\left(y_{i}, r_{y_{i}}\right)\right)^{\frac{n-1}{n}}}{\sum_{i} r_{y_{i}}^{-1} \operatorname{Vol}\left(B\left(y_{i}, r_{y_{i}}\right)\right)} \\
& \leq 10^{2 n+5} \sup _{i} \frac{\operatorname{Vol}\left(B\left(y_{i}, r_{y_{i}}\right)\right)^{\frac{n-1}{n}}}{r_{y_{i}}^{-1} \operatorname{Vol}\left(B\left(y_{i}, r_{y_{i}}\right)\right)} \\
& =10^{2 n+5} \sup _{i}\left(\frac{r_{y_{i}}^{n}}{\operatorname{Vol}\left(B\left(y_{i}, r_{y_{i}}\right)\right)}\right)^{1 / n} .
\end{aligned}
$$

On the other hand, by (3.2),

$$
\operatorname{Vol}\left(B\left(y_{i}, r_{y_{i}}\right)\right) \geq \frac{2}{3} \cdot \frac{r_{y_{i}}^{n}}{r_{1}^{n}} \operatorname{Vol}\left(B\left(y_{i}, r_{1}\right)\right) \geq \frac{4}{3} \cdot \frac{r_{y_{i}}^{n}}{r_{1}^{n}} \operatorname{Vol}\left(B\left(y_{i}, 2 r\right)\right) \geq \frac{4 \delta^{n}}{3} \cdot \frac{r_{y_{i}}^{n}}{r_{0}^{n}} \operatorname{Vol}(B(x, r)) .
$$

Therefore,

$$
\frac{\operatorname{Vol}(\Omega)^{\frac{n-1}{n}}}{\operatorname{Vol}(\partial \Omega)} \leq 10^{2 n+5}\left(\frac{3 r^{n}}{4 \delta^{n} \operatorname{Vol}(B(x, r))}\right)^{1 / n} .
$$

This finishes the proof of the theorem.

It is well known that the isoperimetric inequality above is equivalent to the following Sobolev inequality. 
Corollary 3.8. Under the same assumptions as in the above theorem, we have the following Sobolev inequalities.

$$
\left(\oint_{B(x, r)}|f|^{\frac{n}{n-1}} d g\right)^{\frac{n-1}{n}} \leq C(n) r \oint_{B(x, r)}|\nabla f| d g
$$

and

$$
\left(\oint_{B(x, r)}|f|^{\frac{2 n}{n-2}} d g\right)^{\frac{n-2}{n}} \leq C(n) r^{2} \oint_{B(x, r)}|\nabla f|^{2} d g,
$$

for any $f \in C_{0}^{\infty}(B(x, r))$ and $r \leq r_{0}$.

By setting $V=\nabla L$ for some smooth function $L$ in (2.1), it immediately follows from Theorem 3.7 and Corollary 3.8 that

Corollary 3.9. Suppose that

$$
\text { Ric }+ \text { Hess } L \geq-\lambda g
$$

and

$$
|\nabla L(x)| \leq \frac{K}{d(x, O)^{\alpha}}
$$

for all $x \in \mathbf{M}$, a fixed point $O \in \mathbf{M}$, constants $K \geq 0, \alpha \in[0,1)$, and assume in addition that the noncollapsing condition (2.16) holds if $\alpha>0$. Then there exists an $r_{0}=r_{0}(n, \lambda, K, \alpha, \rho)$ such that for any $r \leq r_{0}$,

(a) $I D_{n}^{*}(B(x, r)) \leq C(n) r$, and

$$
\left(\oint_{B(x, r)}|f|^{\frac{n}{n-1}} d g\right)^{\frac{n-1}{n}} \leq C(n) r \oint_{B(x, r)}|\nabla f| d g,
$$

for any $f \in C_{0}^{\infty}(B(x, r))$.

On the other hand, by a similar argument, we can also show the following

Corollary 3.10. Suppose that

$$
\operatorname{Ric}+\operatorname{Hess} L \geq-\lambda g,
$$

with L satisfying (2.44), i.e.,

$$
|L(y)-L(z)| \leq K_{1} d(y, z)^{\alpha}, \text { and } \sup _{x \in \mathbf{M}, 0 \leq r \leq 1}\left(r^{\beta}\|\nabla L\|_{q, B(x, r)}^{*}\right) \leq K_{2} .
$$

Then there exists an $r_{0}=r_{0}\left(n, \lambda, K_{1}, K_{2}, \alpha, \beta\right)$ such that for any $r \leq r_{0}$,

(a) $I D_{n}^{*}(B(x, r)) \leq C(n) r$, and

(b)

$$
\left(\oint_{B(x, r)}|f|^{\frac{n}{n-1}} d g\right)^{\frac{n-1}{n}} \leq C(n) r \oint_{B(x, r)}|\nabla f| d g,
$$

for any $f \in C_{0}^{\infty}(B(x, r))$.

Note that the non-collapsing condition is not needed in this corollary. In all the results in this section, if $\alpha=0$ in (2.2) , i.e., $|V|$ is bounded, then the non-collapsing condition is not needed. 


\section{Gradient estimates, mean value inequality, heat kernel Bounds And CUT-OFF FUNCTIONS}

In this section, we first state local gradient estimates and mean value inequalities for solutions of the Poisson equation and the heat equation. The general method of proof is more or less standard, using the Sobolev inequality in the previous section and Moser's iteration. However, comparing with the standard case, we need to deal with the Ricci curvature term in a different manner. For this reason, a proof in the Poisson equation case is presented in detail in Appendix I while the case for the heat equation is omitted.

These results allow us to establish the Gaussian upper and lower bounds of the heat kernel. Finally, we use the heat kernel to construct a good cut-off function which will be needed in the proofs of the main results.

In the following context, we denote by

$$
\|f\|_{q, B(x, r)}^{*}=\left(\oint_{B(x, r)}|f|^{q}\right)^{1 / q} .
$$

Theorem 4.1 (Elliptic gradient estimate). Assume that (2.1), (2.2) and (2.16) hold, i.e.,

$$
\operatorname{Ric}+\frac{1}{2} \mathcal{L}_{V} g \geq-\lambda g ; \quad|V|(y) \leq \frac{K}{d(y, O)^{\alpha}}, \alpha \in[0,1) ; \quad \operatorname{Vol}(B(x, 1)) \geq \rho .
$$

Then there exists a positive constant $r_{0}=r_{0}(n, \lambda, K, \alpha, \rho)$ such that, for any $x \in \mathbf{M}$, $0<r \leq r_{0}$ and smooth functions $u$ and $f$ satisfying the equation

$$
\Delta u=f, \text { in } B(x, r) \text {, }
$$

we have

$$
\sup _{B\left(x, \frac{1}{2} r\right)}|\nabla u|^{2} \leq C(n, \lambda, K, \alpha, \rho) r^{-2}\left[\left(\|u\|_{2, B(x, r)}^{*}\right)^{2}+\left(\|f\|_{2 q, B(x, r)}^{*}\right)^{2}\right]
$$

for any $q>n / 2$. Moreover

$$
\sup _{B\left(x, \frac{1}{2} r\right)} u^{2} \leq C(n, \lambda, K, \alpha, \rho)\left[\left(\|u\|_{2, B(x, r)}^{*}\right)^{2}+\left(\|f\|_{q, B(x, r)}^{*}\right)^{2}\right] .
$$

In particular if $\alpha=0$, then the conclusions hold without the non-collapsing condition.

Proof. See Appendix I.

By a similar argument, we get

Corollary 4.2. Suppose that

$$
\text { Ric }+\operatorname{Hess} L \geq-\lambda g
$$

with L satisfying (2.44), i.e.,

$$
|L(y)-L(z)| \leq K_{1} d(y, z)^{\alpha}, \text { and } \sup _{x \in \mathbf{M}, 0 \leq r \leq 1}\left(r^{\beta}\|\nabla L\|_{q, B(x, r)}^{*}\right) \leq K_{2} .
$$

Then there exists a constant $r_{0}=r_{0}\left(n, \lambda, K_{1}, K_{2}, \alpha, \beta\right)$, such that for any $x \in \mathbf{M}, 0<r \leq$ $r_{0}$ and smooth functions $u$ and $f$ satisfying the equation

$$
\Delta u=f
$$


in $B(x, r)$, we have

$$
\sup _{B\left(x, \frac{1}{2} r\right)}|\nabla u|^{2} \leq C\left(n, \lambda, K_{1}, K_{2}, \alpha, \beta\right) r^{-2}\left[\left(\|u\|_{2, B(x, r)}^{*}\right)^{2}+\left(\|f\|_{2 q, B(x, r)}^{*}\right)^{2}\right],
$$

for any $q>n / 2$. Moreover,

$$
\sup _{B\left(x, \frac{1}{2} r\right)} u^{2} \leq C\left(n, \lambda, K_{1}, K_{2}, \alpha, \beta\right)\left[\left(\|u\|_{2, B(x, r)}^{*}\right)^{2}+\left(\|f\|_{q, B(x, r)}^{*}\right)^{2}\right] .
$$

As mentioned at the beginning of the section, the same argument also implies the corresponding result for the heat equation:

Theorem 4.3 (Parabolic gradient estimate). Suppose the same conditions as in Theorem 4.1 hold. Then there exists a positive constant $r_{0}=r_{0}(n, \lambda, K, \alpha, \rho)$ such that, for any $x \in \mathbf{M}, 0<r \leq r_{0}$ and smooth functions $u$ and $f$ satisfying the heat equation

$$
\Delta u(x, t)-\partial_{t} u(x, t)=f(x)
$$

in $Q(x, t, r)=B(x, r) \times\left[t-r^{2}, t\right]$, we have

$$
\sup _{Q\left(x, t, \frac{1}{2} r\right)}|\nabla u|^{2} \leq C(n, \lambda, K, \alpha, \rho) r^{-2}\left[\left(\|u\|_{2, Q(x, t, r)}^{*}\right)^{2}+\left(\|f\|_{2 q, B(x, r)}^{*}\right)^{2}\right],
$$

for any $q>\frac{n}{2}$. Moreover,

$$
\sup _{Q\left(x, t, \frac{1}{2} r\right)} u^{2} \leq C(n, \lambda, K, \alpha, \rho)\left[\left(\|u\|_{2, Q(x, t, r)}^{*}\right)^{2}+\left(\|f\|_{q, B(x, r)}^{*}\right)^{2}\right] .
$$

Since the Sobolev inequality, volume comparison, volume non-collapsing, and gradient bound holds, it is well known that the following heat kernel bounds hold. The proof is omitted.

Theorem 4.4 (Heat kernel bounds). Under the same conditions as in Theorem 4.1, for $G=G(x, t ; y, 0)$, the heat kernel on the manifold $\mathbf{M}$, the following bounds hold for some positive constants $C_{i}=C_{i}(n, \lambda, K, \alpha, \rho), i=1, \cdots, 4$.

$$
\begin{gathered}
C_{1} t^{-\frac{n}{2}} e^{\frac{-C_{2} d^{2}(x, y)}{t}} \leq G(x, t ; y, 0) \leq C_{3} t^{-\frac{n}{2}} e^{-\frac{d^{2}(x, y)}{C_{4} t}}, \forall x, y \in \mathbf{M}, \text { and } 0<t \leq 1 . \\
\left|\nabla_{x} G(x, t ; y, 0)\right| \leq C_{3} t^{-\frac{n+1}{2}} e^{-\frac{d^{2}(x, y)}{C_{4} t}}, \forall x, y \in \mathbf{M}, \text { and } 0<t \leq 1 . \\
\left|\partial_{t} G(x, t ; y, 0)\right| \leq C_{3} t^{-\frac{n+2}{2}} e^{-\frac{d^{2}(x, y)}{C_{4} t}}, \forall x, y \in \mathbf{M}, \text { and } 0<t \leq 1 .
\end{gathered}
$$

Similar to Corollary 3.2 in [PeWe2], we may also use the Sobolev inequality in Corollary 3.8 to prove the following maximum principle. Since the proof is identical, it is skipped.

Theorem 4.5 (Maximum principle). Under the same conditions as in Theorem 4.1, there is an $r_{0}=r_{0}(n, \lambda, K, \alpha, \rho)$ so that for any $r \leq r_{0}$ and function $u$ satisfying

$$
\Delta u \geq f, \text { in } B(x, r),
$$

we have

$$
\sup _{B(x, r)} u \leq \sup _{\partial B(x, r)} u+C(n) r^{2}\|f\|_{q, B(x, r)}^{*},
$$

where $q>\frac{n}{2}$. 
To prove the volume convergence and cone rigidity for Gromov-Hausdorff limits, another important tool is to construct an appropriate cut-off function (see e.g., Theorem 6.33 in [ChCo1]). Since we have obtained Sobolev inequality (3.7) and gradient estimate (Theorem 4.1), we may wish to follow the proof of Theorem 6.33 in ChCo1 to show the existence of the cut-off function in the following lemma. However, the proof may be quite technical. Instead, we adapt a method in [BZ and an covering argument in CN] (see also [Bam]) to construct the cut-off function through the heat kernel estimates in Theorem 4.4. This method may have a broader application.

Lemma 4.6 (Cut-off function). Suppose again that the basic assumption (2.1), (2.2) and the volume non-collapsing condition (2.16) hold. Then for any $x_{0} \in \mathbf{M}$ and $0<r_{0} \leq 5$, there exists a function $\phi \in C^{2}(\mathbf{M})$ satisfying

(1) $0 \leq \phi \leq 1$ everywhere.

(2) $\operatorname{supp} \phi \subset \subset B\left(x_{0}, 1.9 r_{0}\right)$.

(3) $\phi=1$ in $B\left(x_{0}, 1.1 r_{0}\right)$.

(4) $|\nabla \phi|<C(n, \lambda, K, \alpha, \rho) r_{0}^{-1}$ and $|\Delta \phi|<C(n, \lambda, K, \alpha, \rho) r_{0}^{-2}$.

Remark 4.7. Note that in (2) and (3) above, 1.9 and 1.1 are of nothing special. In fact, we may choose any numbers.

Proof. The proof consists of two steps.

Step 1: We first show that there exists a constant $\delta=\delta(n, \lambda, K, \alpha, \rho)>0$ such that for any point $x_{0} \in \mathbf{M}$ and constant $r_{0}>0$, there exists a function $\psi \in C^{\infty}(\mathbf{M})$ satisfying:

(a) $0 \leq \psi<1$ everywhere.

(b) $\psi>\delta$ in $B\left(x_{0}, \delta r_{0}\right)$.

(c) $\psi=0$ in $\mathbf{M} \backslash B\left(x_{0}, r_{0}\right)$.

(d) $|\nabla \psi|<r_{0}^{-1}$ and $|\Delta \psi|<r_{0}^{-2}$ everywhere.

Consider the function $G(x)=G\left(x, A r_{0}^{2} ; x_{0}, 0\right)$, where $G\left(x, t ; x_{0}, 0\right)$ denotes the heat kernel, and $A$ is a constant to be determined. From Theorem 4.4, we have

$$
C_{1}\left(A r_{0}^{2}\right)^{-\frac{n}{2}} \leq G\left(x_{0}\right) \leq C_{3}\left(A r_{0}^{2}\right)^{-\frac{n}{2}}
$$

If $d\left(x, x_{0}\right) \geq r_{0}$, then

$$
G(x) \leq C_{3}\left(A r_{0}^{2}\right)^{-\frac{n}{2}} e^{-\frac{d^{2}\left(x, x_{0}\right)}{C_{4} A r_{0}^{2}}} \leq C_{3}\left(A r_{0}^{2}\right)^{-\frac{n}{2}} e^{-\frac{1}{A C_{4}}} .
$$

Thus by choosing $A=\frac{1}{C_{4} \ln \left(2 C_{3} / C_{1}\right)}$, we have

$$
G(x) \leq \frac{1}{2} C_{1}\left(A r_{0}^{2}\right)^{-\frac{n}{2}}, \text { in } \mathbf{M} \backslash B\left(x_{0}, r_{0}\right) .
$$

Also in $B\left(x_{0}, r_{0}\right)$,

$$
G(x) \leq G\left(x_{0}\right)+|\nabla G| d\left(x, x_{0}\right) \leq C_{3}\left[\left(A r_{0}^{2}\right)^{-\frac{n}{2}}+\left(A r_{0}^{2}\right)^{-\frac{n+1}{2}} r_{0}\right]=\left(1+A^{-1 / 2}\right) C_{3}\left(A r_{0}^{2}\right)^{-\frac{n}{2}}
$$

Let

$$
\hat{\psi}(x)=\max \left\{G(x)-0.6 C_{1}\left(A r_{0}^{2}\right)^{-\frac{n}{2}}, 0\right\} .
$$

Then supp $\hat{\psi} \subset \subset B\left(x_{0}, r_{0}\right)$. Moreover, when $\hat{\psi}>0$, (4.2) implies that

$$
\hat{\psi}(x) \leq\left[\left(1+A^{-1 / 2}\right) C_{3}-0.6 C_{1}\right]\left(A r_{0}^{2}\right)^{-\frac{n}{2}},
$$


in $B\left(x_{0}, r_{0}\right)$, and Theorem 4.4 gives

$$
|\nabla \hat{\psi}|=|\nabla G(x)| \leq C_{3}\left(A r_{0}^{2}\right)^{-\frac{n+1}{2}} \text {, and }|\Delta \hat{\psi}|=|\Delta G|=\left|\partial_{t} G\right| \leq C_{3}\left(A r_{0}^{2}\right)^{-\frac{n+2}{2}} .
$$

On the other hand, from (4.1), we have $\hat{\psi}\left(x_{0}\right) \geq 0.4 C_{1}\left(A r_{0}^{2}\right)^{-\frac{n}{2}}$. Thus, setting $\hat{\delta}=$ $\frac{0.4 C_{1}}{\left(1+A^{-1 / 2}+A^{-1}\right) C_{3}}$ yields that in $B\left(x_{0}, \hat{\delta} r_{0}\right)$,

$$
\begin{aligned}
\hat{\psi}(x) \geq \hat{\psi}\left(x_{0}\right)-|\nabla \hat{\psi}|\left(x^{\prime}\right) d\left(x, x_{0}\right) & \geq 0.4 C_{1}\left(A r_{0}^{2}\right)^{-\frac{n}{2}}-C_{3}\left(A r_{0}^{2}\right)^{-\frac{n+1}{2}} \hat{\delta} r_{0} \\
& =\hat{\delta}\left[\left(1+A^{-1}\right) C_{3}\right]\left(A r_{0}^{2}\right)^{-\frac{n}{2}}
\end{aligned}
$$

Combining (4.3), (4.4) and (4.5), we see that function $\tilde{\psi}=\frac{1}{\left(1+A^{-1}\right) C_{3}}\left(A r_{0}^{2}\right)^{\frac{n}{2}} \hat{\psi}$ satisfies

$$
\begin{gathered}
0 \leq \tilde{\psi} \leq 1 \text { in } B\left(x_{0}, r_{0}\right), \quad \tilde{\psi} \geq \hat{\delta} \text { in } B\left(x_{0}, \hat{\delta} r_{0}\right), \\
|\nabla \tilde{\psi}| \leq r_{0}^{-1} \text {, and }|\Delta \tilde{\psi}| \leq r_{0}^{-2} .
\end{gathered}
$$

Now, let $\psi=\tilde{\psi}^{3}$. Then $\psi$ is $C^{2}$ differentiable and still satisfies (a)-(d).

Step 2: From step 1 and using the same covering argument as in [CN] and also Lemma 6.13 in [Bam, we can prove the existence of the cut-off function $\phi$ in the lemma. For a fixed small $\epsilon>0$, let $N$ be the smallest integer such that $N$ balls of radius $\epsilon \delta r_{0}$ cover $B\left(x_{0}, 1.1 r_{0}\right)$. We denote these balls by $B\left(p_{i}, \epsilon \delta r_{0}\right), i=1, \ldots N$. By the volume comparison theorem and noncollapsing condition, $N$ is uniformly bounded. Let $\psi_{i}$ be the cut-off function obtained in step1 for the ball $B\left(p_{i}, \epsilon r_{0}\right)$. Let $\eta$ be a smooth function from $[0, \infty)$ to $[0,1]$ such that $\eta(s)=1$ when $s \geq 1$. Then it is easy to check the function

$$
\psi=\eta\left(\Sigma_{i=1}^{N} \delta^{-1} \psi_{i}\right)
$$

is the desired cut-off function when $\epsilon<0.1$.

\section{Segment inequality, exCess estimate And $\epsilon$-SPlitting map}

In this section, we will build the three essential tools: segment inequality, excess estimate and $\epsilon$-splitting map for proving certain degeneration results for Riemannian manifolds under the modified Ricci curvature bound. From now on, unless otherwise stated, we always assume that (2.1), (2.2) and (2.16) hold, i.e.,

$$
\operatorname{Ric}+\frac{1}{2} \mathcal{L}_{V} g \geq-\lambda g, \quad|V|(y) \leq \frac{K}{d(y, O)^{\alpha}}, \quad \alpha \in[0,1)
$$

and

$$
\operatorname{Vol}(B(y, 1)) \geq \rho>0, \quad \forall y \in \mathbf{M} .
$$

Note that this condition allows gradient Ricci solitons whose potential function $L$ has bounded gradient. It also covers the case when the Bakry-Émery Ricci curvature has a lower bound and the potential function $L$ has bounded gradient. In this case, F. Wang and X.H. Zhu WZ] have obtained, among other things, degeneration results using weighted manifold approach. If both $|\nabla L|$ and $\Delta L$ are bounded, then, according to Tian and Z.L. Zhang [TZ, one can use a conformal transform to convert the metric to a new one with lower Ricci bound. Then the degeneration results follow from Cheeger and Colding's work. 
In proving the degeneration results, we will follow the road maps by Cheeger and Colding.

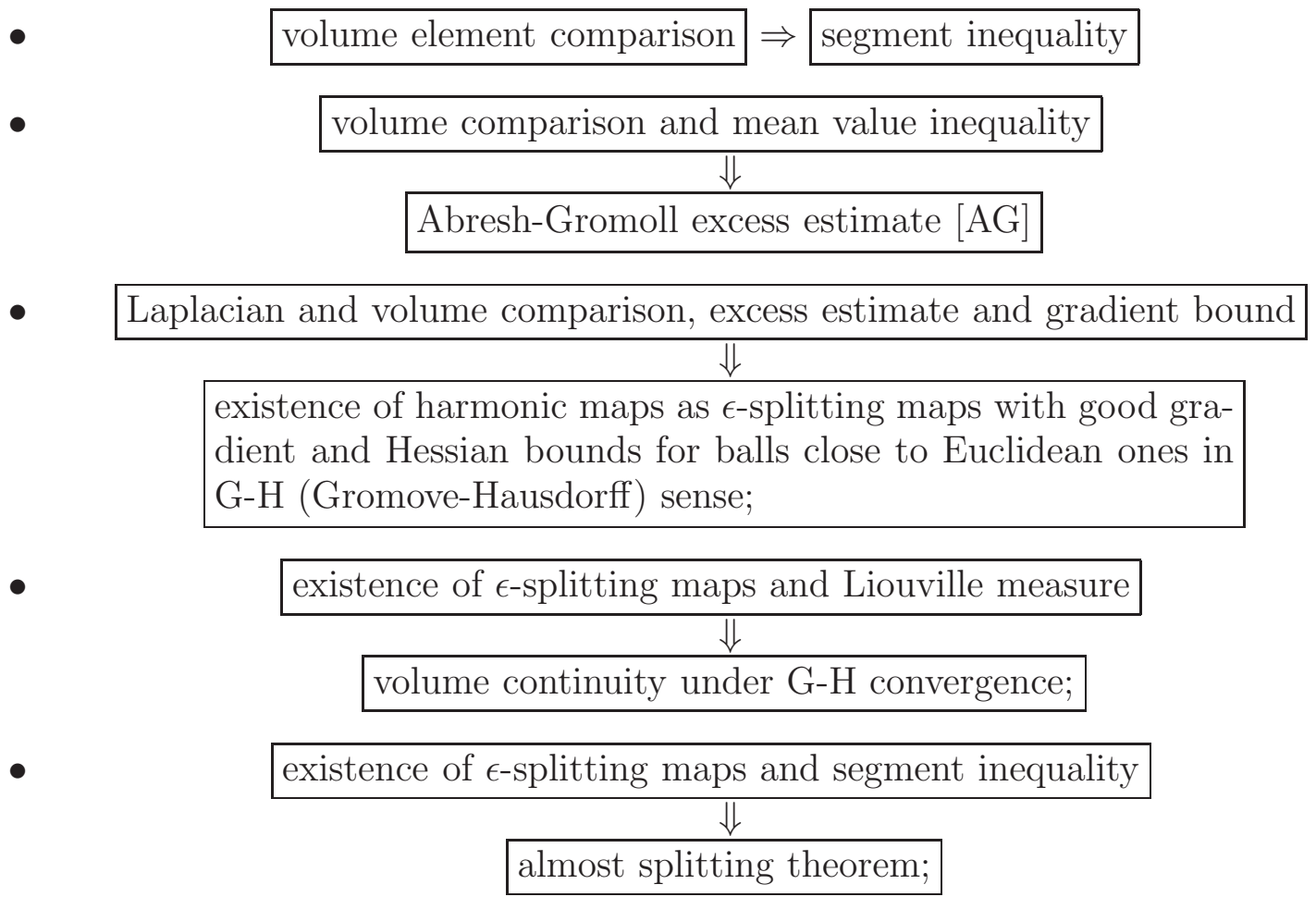

- existence of $\epsilon$-splitting maps, segment inequality and almost splitting theorem
$\Downarrow$
existence of metric cones.

Identical proofs will be omitted. Proof that needs moderate modifications will be presented in the appendices. Proofs that requires new ingredients will be presented in the main text.

Given a smooth function $f \geq 0$, let

$$
\mathcal{F}_{f}\left(x_{1}, x_{2}\right)=\inf _{\gamma} \int_{0}^{d\left(x_{1}, x_{2}\right)} f(\gamma(s)) d s
$$

where the infimum is taken over all minimal geodesics from $x_{1}$ to $x_{2}$.

We first prove a similar segment inequality as in ChCo1. The proof is essentially the same as the proof of Theorem 2.11 in [ChCo1, where the key ingredient in the proof is the comparison of the volume elements, which in our case is (2.14). For completeness, we present the proof in Appendix II. From Theorem 2.2, we see that (2.14) only requires assumption (5.1). More explicitly, we have

Theorem 5.1 (Segment inequality). Let $F_{f}\left(x_{1}, x_{2}\right)$ be the function defined in (5.3). Suppose that (5.1) is satisfied. Then for any $r>0$ and measurable sets $A_{1}, A_{2} \subseteq B(x, r)$, we have

$$
\int_{A_{1} \times A_{2}} \mathcal{F}_{f}\left(x_{1}, x_{2}\right) \leq C(n, \lambda, K, \alpha)\left(\operatorname{Vol}\left(A_{1}\right)+\operatorname{Vol}\left(A_{2}\right)\right) r \int_{B(x, 3 r)} f d g .
$$


The proof can be found in Appendix II.

Next, we deal with the excess estimate. Fix $q_{+}$and $q_{-}$in $\mathbf{M}$, and define the excess function on $\mathbf{M}$ as

$$
e(y)=d\left(y, q_{+}\right)+d\left(y, q_{-}\right)-d\left(q_{+}, q_{-}\right) .
$$

For any $x \in \mathbf{M}$, if we denote by

$$
b_{ \pm}(y)=d\left(y, q_{ \pm}\right)-d\left(x, q_{ \pm}\right)
$$

then

$$
b_{+}(y)+b_{-}(y)=e(y)-e(x) .
$$

Since we have the heat kernel bounds, the following mean value type inequality can be obtained routinely by using Duhamel's formula. One may imitate the proof of Corollary 4.8 in [ZZ, which is a version of Theorem 8.18 in [GT] and Lemma 2.1 of Colding and Naber [CN].

Lemma 5.2. Let $u(x)$ be a nonnegative function satisfying

$$
\Delta u \leq \xi(x)
$$

where $\xi(x) \geq 0$ is a smooth function.

If (5.1) and (5.2) are satisfied, then for any $q>\frac{n}{2}$, there exists a constant $C=$ $C(n, \lambda, K, \alpha, \rho)$ such that

$$
\oint_{B(x, r)} u(y) d y \leq C\left(u(x)+r^{2}\|\xi\|_{q, B(x, r)}^{*}\right)
$$

holds for any $x \in M$ and $0<r \leq 1$.

More generally, we have

$$
\oint_{B(x, r)} u(y) d y \leq C\left(\inf _{B(x, r)} u(\cdot)+r^{2}\|\xi\|_{q, B(x, r)}^{*}\right) .
$$

Applying the above lemma to the excess function yields the following excess estimate. In the context below, we will use $\Psi\left(\epsilon_{1}, \epsilon_{2}, \cdots, \epsilon_{k} \mid c_{1}, c_{2}, \cdots, c_{N}\right)$ to denote a nonnengative function such that for any fixed $c_{1}, c_{2}, \cdots, c_{N}$,

$$
\lim _{\epsilon_{1}, \epsilon_{2}, \cdots, \epsilon_{k} \rightarrow 0} \Psi=0 .
$$

Theorem 5.3 (Excess estimate). Suppose that (5.1) and (5.2) are satisfied. Assume in addition that

$$
d\left(x, q_{ \pm}\right)=O\left(\lambda^{-\frac{1}{2}}\right)
$$

and

$$
e(x) \leq \epsilon R,
$$

for some $R \leq 1$. Then there exists $a \Psi=\Psi(\lambda, K, \epsilon \mid n, R, \alpha, \rho)$ such that

$$
e(y) \leq \Psi R, \text { in } B(x, R) .
$$

Proof. The proof, which is different from the original one in [AG], is inspired by Remark 2.9 [CN. Note that by (5.5),

$$
\Delta e(y)=\Delta b_{+}+\Delta b_{-} \leq \frac{n-1}{d\left(y, q_{+}\right)}+\frac{n-1}{d\left(y, q_{-}\right)}+\psi_{+}+\psi_{-},
$$


where by Proposition 2.1,

$$
\psi_{ \pm}=\left(\Delta d\left(y, q_{ \pm}\right)-\frac{n-1}{d\left(y, q_{ \pm}\right)}\right)_{+} \leq \frac{\lambda}{3} d\left(y, q_{ \pm}\right)+|V|(y)+\frac{C(\alpha) K}{d^{\alpha}\left(y, q_{ \pm}\right)} .
$$

According to (5.12), Lemma 2.4 and Lemma 5.2, for $y \in B(x, R)$, we have

$$
\begin{aligned}
\oint_{B(x, R)} e(y) d y & \leq C\left(\inf _{B(x, R)} u(\cdot)+\frac{2(n-1) R^{2}}{\lambda^{-\frac{1}{2}}-R}+R^{2}\left\|\psi_{+}\right\|_{q, B(x, R)}^{*}+R^{2}\left\|\psi_{-}\right\|_{q, B(x, R)}^{*}\right) \\
& \leq C\left(\epsilon R+\frac{2(n-1) R^{2}}{\lambda^{-\frac{1}{2}}-R}+\lambda R^{2}\left(\lambda^{-\frac{1}{2}}+R\right)+\frac{K R^{2}}{\left(\lambda^{-\frac{1}{2}}-R\right)^{\alpha}}+R^{2-\alpha} K\right) \\
& =\Psi_{1} R .
\end{aligned}
$$

Therefore, by using Theorem 2.2 , for any $y \in B\left(x,\left(1-\Psi_{2}\right) R\right)$, we have

$$
\begin{aligned}
\oint_{B\left(y, \Psi_{2} R\right)} e(z) d z & \leq \frac{\operatorname{Vol}(B(x, R))}{\operatorname{Vol}\left(B\left(y, \Psi_{2} R\right)\right)} \oint_{B(x, R)} e(z) d z \\
& \leq \frac{\operatorname{Vol}(B(y, 2 R))}{\operatorname{Vol}\left(B\left(y, \Psi_{2} R\right)\right)} \Psi_{1} R \\
& \leq \frac{\Psi_{1}}{\Psi_{2}^{n}} R .
\end{aligned}
$$

Hence, if we choose $\Psi_{2}=\Psi_{1}^{\frac{1}{n+1}}$, then the above inequality becomes

$$
\oint_{B\left(y, \Psi_{2} R\right)} e(z) d z \leq \Psi_{2} R
$$

It implies that there exists a $z \in B\left(y, \Psi_{2} R\right)$ such that

$$
e(z) \leq \Psi_{2} R,
$$

and hence by mean value theorem

$$
e(y) \leq e(z)+|\nabla e| d(y, z) \leq \Psi_{2} R+2 \Psi_{2} R=3 \Psi_{2} R .
$$

Since $y$ is an arbitrary point in $B\left(x,\left(1-\Psi_{2}\right) R\right)$, applying mean value theorem one more time for points in $B(x, R) \backslash B\left(x,\left(1-\Psi_{2}\right) R\right)$ completes the proof of the theorem.

Let $h_{+}$and $h_{-}$be harmonic functions in $B(x, R)$ such that $\left.h_{ \pm}\right|_{\partial B(x, R)}=b_{ \pm}$. Since the excess estimate (5.11) has been established, following the method in section 6 of [ChCo1] (see also section 9 in [Ch]), it is not hard to show that

Lemma 5.4. Under the basic assumption (5.1), (5.2), suppose (5.9) and (5.10) hold i.e. $d\left(x, q_{ \pm}\right)=O\left(\lambda^{-\frac{1}{2}}\right)$ and $e(x) \leq \epsilon R$. Then there exists an $r_{0}=r_{0}(n, \lambda, K, \alpha, \rho)$ such that for any $R \leq r_{0}$ and some $\Psi=\Psi(\lambda, K, \epsilon \mid n, R, \alpha, \rho)$, we have

(1) $\left|h_{ \pm}-b_{ \pm}\right| \leq \Psi R$ in $B(x, R)$;

(2) $\oint_{B(x, R)}\left|\nabla b_{ \pm}-\nabla h_{ \pm}\right|^{2} \leq \Psi$;

(3) $\oint_{B\left(x, \frac{1}{2} R\right)}\left|\nabla^{2} h_{ \pm}\right|^{2} \leq \Psi R^{-2}$. 
Proof of (1): Since $\Delta h_{ \pm}=0$, by Proposition 2.1, we have

$$
\Delta\left(h_{ \pm}-b_{ \pm}\right) \geq-\psi_{ \pm}
$$

where $\psi_{ \pm}=\frac{\lambda}{3} d\left(\cdot, q_{ \pm}\right)+|V|(\cdot)+\frac{C(\alpha) K}{d\left(\cdot, q_{ \pm}\right)^{\alpha}}$.

Thus, Theorem 4.5 implies that

$$
\begin{aligned}
h_{ \pm}-b_{ \pm} & \leq C(n) R^{2}\left\|\psi_{ \pm}\right\|_{q, B(x, R)}^{*} \\
& \leq C(n, \lambda, K, \alpha, \rho) R^{2}\left[\lambda\left(\lambda^{-\frac{1}{2}}+R\right)+K R^{-\alpha}+K\left(\lambda^{-\frac{1}{2}}-R\right)^{-\alpha}\right] \\
& =\Psi R .
\end{aligned}
$$

For the lower bounds, notice that $b_{+}(y)+b_{-}(y)=e(y)-e(x)$. It follows from Theorem 5.3 that

$$
-\epsilon R \leq b_{+}(y)+b_{-}(y) \leq \Psi R,
$$

which in turn, by the maximal principle, implies that

$$
h_{+}(y)+h_{-}(y) \geq-\epsilon R .
$$

Combining (5.13), (5.14) and (5.15), we derive

$$
b_{+} \leq-b_{-}+\Psi R \leq-h_{-}+2 \Psi R \leq h_{+}+2 \Psi R+\epsilon R,
$$

i.e.,

$$
h_{+}-b_{+} \geq-\Psi R \text {. }
$$

A lower bound for $h_{-}-b_{-}$can be obtained in a similar way.

Proof of (2): We only prove the result for $h_{+}$, the proof for $h_{-}$is similar. By (1), Theorem 2.2 and (5.13), we get

$$
\begin{aligned}
\oint_{B(x, R)}\left|\nabla h_{+}-\nabla b_{+}\right|^{2} & =\oint_{B(x, R)}\left(h_{+}-b_{+}\right) \Delta b_{+} \\
& \leq \Psi R\left|\oint_{B(x, R)}\left(\Delta b_{+}\right)_{-}-\left(\Delta b_{+}\right)_{+}\right|+2 \Psi R \oint_{B(x, R)}\left(\Delta b_{+}\right)_{+} \\
& \leq\left(\frac{\operatorname{Vol}(\partial B(x, R))}{\operatorname{Vol}(B(x, R))}\right) \Psi R+2 \Psi R \oint_{B(x, R)} \psi_{+} \\
& \leq \Psi .
\end{aligned}
$$

Proof of (3): Again, we only present the proof for $h_{+}$. By Lemma 4.6, we may choose a cut-off function $\phi$ so that

$$
\operatorname{supp} \phi \subset B(x, R),\left.\quad \phi\right|_{B(x, R / 2)}=1, \quad|\nabla \phi|^{2}+|\Delta \phi| \leq \frac{C(n, \lambda, K, \alpha, \rho)}{R^{2}} .
$$

By the Bochner's formula,

$$
\begin{aligned}
\oint_{B(x, R)} 2 \phi\left|\nabla^{2} h_{+}\right|^{2} & =\oint_{B(x, R)} \phi\left(\Delta\left|\nabla h_{+}\right|^{2}-2 \operatorname{Ric}\left(\nabla h_{+}, \nabla h_{+}\right)\right) \\
& \leq \oint_{B(x, R)} \phi\left(\Delta\left(\left|\nabla h_{+}\right|^{2}-1\right)+2 \nabla_{i} V_{j} \nabla_{i} h_{+} \nabla_{j} h_{+}+2 \lambda\left|\nabla h_{+}\right|^{2}\right) .
\end{aligned}
$$


Notice that

$$
\begin{aligned}
\oint_{B(x, R)} 2 \phi \nabla_{i} V_{j} \nabla_{i} h_{+} \nabla_{j} h_{+} & =\oint_{B(x, R)}-2 \nabla_{i} \phi V_{j} \nabla_{i} h_{+} \nabla_{j} h_{+}-2 \phi V_{j} \nabla_{i} h_{+} \nabla_{i} \nabla_{j} h_{+} \\
& \leq \oint_{B(x, R)} 2|\nabla \phi||V|\left|\nabla h_{+}\right|^{2}+\oint_{B(x, R)} \phi\left|\nabla^{2} h_{+}\right|^{2}+\phi|V|^{2}\left|\nabla h_{+}\right|^{2} .
\end{aligned}
$$

Thus, (5.16) becomes

$$
\oint_{B(x, R)} \phi\left|\nabla^{2} h_{+}\right|^{2} \leq \oint_{B(x, R)}\left(\left|\nabla h_{+}\right|^{2}-1\right) \Delta \phi+2|\nabla \phi||V|\left|\nabla h_{+}\right|^{2}+\phi|V|^{2}\left|\nabla h_{+}\right|^{2}+2 \lambda\left|\nabla h_{+}\right|^{2} .
$$

By the gradient estimate in Theorem 4.1 and the assumed bound on $V$, we see that

$$
\left|\nabla h_{+}\right|^{2} \leq C R^{-2}\left(\left\|h_{+}\right\|_{2, B(x, 2 R)}^{*}\right)^{2} \leq C R^{-2}\left(\left\||| b_{+} \mid+\Psi R\right\|_{2, B(x, 2 R)}^{*}\right)^{2} \leq C(n, \lambda, K, \alpha, \rho, R) .
$$

Then, using the conclusion in (2), Lemma 2.4 and (5.18) for the RHS of (5.17) yields

$$
\oint_{B(x, R)} \phi\left|\nabla^{2} h_{+}\right|^{2} \leq \Psi R^{-2}
$$

which finishes the proof of (3) due to the fact that $\left.\phi\right|_{B\left(x, \frac{1}{2} R\right)}=1$ and the volume comparison theorem 2.2 .

Lemma 5.4 together with the segment inequality implies, as in Theorem 6.62 [ChCo1], Theorem 5.5. If (5.1), (5.2), (5.9) and (5.10) hold, then for some $r_{0}=r_{0}(n, \lambda, K, \alpha, \rho)$ and any $R \leq r_{0}$, there exists a metric space $X$ such that for some ball $B\left((0, \tilde{x}), \frac{1}{4} R\right) \subseteq$ $\mathbb{R} \times X$, with the product metric, we have

$$
d_{G H}\left(B\left(x, \frac{1}{4} R\right), B\left((0, \tilde{x}), \frac{1}{4} R\right)\right) \leq \Psi .
$$

The above theorem amounts to the following almost splitting theorem, as in Theorem 6.64 ChCo1.

Theorem 5.6 (Almost splitting). Let $\left(\mathbf{M}_{i}, d_{i}\right) \stackrel{d_{G H}}{\longrightarrow}(Y, d)$, where $d_{i}$ is the distance function induced by the Riemaniann metric $g_{i}$ on $\mathbf{M}$. Suppose that

$$
\operatorname{Ric}_{\mathbf{M}_{i}}+\frac{1}{2} \mathcal{L}_{V_{i}} g_{\mathbf{M}_{i}} \geq-\lambda_{i} g_{\mathbf{M}_{i}},\left|V_{i}\right|\left(y_{i}\right) \leq \frac{K_{i}}{d_{i}^{\alpha}\left(y_{i}, O_{i}\right)}, \text { and } \operatorname{Vol}\left(B\left(y_{i}, 1\right)\right) \geq \rho,
$$

where $O_{i}$ is a fixed point in $\mathbf{M}_{i}, y_{i}$ is an arbitrary point in $\mathbf{M}_{i}$, and $\lambda_{i}, K_{i} \rightarrow 0$ as $i \rightarrow \infty$.

If $Y$ contains a line, then $Y$ splits as an isometric product, $\mathbb{R} \times X$ for some metric space $X$.

Finally, Lemma 5.4 also yields, according to [Co (see also [ChCo1]), the existence of a so called $\epsilon$-splitting map (see also Definition 1.6 and Lemma 1.7 in [ChNa]).

Definition 5.7. A map $h=\left(h_{1}, h_{2}, \cdots, h_{k}\right): B(x, r) \rightarrow \mathbb{R}^{k}$ is an $\epsilon$-splitting map, if (1) Each $h_{i}, i=1, \cdots, k$, is a harmonic function;

(2) $|\nabla h| \leq 1+\epsilon$; 
(3) $\oint_{B(x, r)}\left|<\nabla h_{i}, \nabla h_{j}>-\delta_{i j}\right|^{2} \leq \epsilon^{2}, \forall i, j ;$
(4) $r^{2} \oint_{B(x, r)}\left|\nabla^{2} h_{i}\right| \leq \epsilon^{2}, \forall i$.

Lemma 5.8. Suppose that (5.1) and (5.2) are satisfied, and

$$
d_{G H}\left(B\left(x, \lambda^{-1}\right), \underline{B}\left(0, \lambda^{-1}\right)\right) \leq \epsilon .
$$

Then for any $R \leq 1$, there exists a $\Psi$-splitting map $h=\left(h_{1}, h_{2}, \cdots, h_{n}\right): B(x, R) \rightarrow \mathbb{R}^{n}$ for some $\Psi=\Psi(\lambda, K, \epsilon \mid n, R, \alpha, \rho)$. Moreover, in the Gromov-Hausdorff sence, $h_{i}$ and $\pi_{i}$ are $\Psi$ close.

Here $\underline{B}(0, L)$ denotes the ball centered at the origin with radius $L$ in $\mathbb{R}^{n}$, and $\pi_{i}$ is the $i$-th coordinate function on $\mathbb{R}^{n}$.

Remark 5.9. Here we recall the idea of the proof of the lemma in the papers cited above. If $d_{G H}\left(B\left(x, \lambda^{-1}\right), \underline{B}\left(0, \lambda^{-1}\right)\right) \leq \epsilon$, then there exists a $2 \epsilon$ isometry $f: \underline{B}\left(0, \lambda^{-1}\right) \rightarrow B\left(x, \lambda^{-1}\right)$. Thus, for any $y \in B\left(x, \lambda^{-1}\right)$, there is a point $y \in \underline{B}\left(0, \lambda^{-1}\right)$ so that $d(f(y), y) \leq 2 \epsilon$. From this $f$, one can easily show that a map formed by certain Buseman functions is a $4 \epsilon$ isometry. Then one can use harmonic functions with the same boundary value on a ball as the Buseman functions to form a $6 \epsilon$ isometry. Then Lemma 5.4 shows this harmonic map satisfies the conditions in definition 5.7. Hence it is an $\epsilon$ splitting.

In the above lemma, $h_{i}$ and $\pi_{i}$ being $\Psi$ close in Gromov-Hausdorff sense means that for any $y \in B\left(x, \lambda^{-1}\right)$, we have $\left|h_{i}(y)-\pi_{i}(\underline{y})\right| \leq \Psi$.

Remark 5.10. If $\alpha=0$ in condition (5.1), then the noncollapsing condition (5.2) is not necessary for all conclusions in this section.

\section{Main theorems: Volume CONTINuity, Metric CONE, Size of Singular SeT}

In this section, we present the volume convergence and volume continuity theorems and prove that any tangent cone of a Gromov-Hausdorff limit under conditions (5.1) and (5.2) is a metric cone.

First, we state the volume convergence and volume continuity theorems. Under the assumption that Ricci curvature bounded from below, the volume continuity was first proved by Colding [Co]. Here we adapt a similar argument as in Theorems 9.31 and 9.40 in [Ch], which essentially uses Lemma 5.8 and the segment inequality. With the preparation in the previous section, the remaining proof now is the same as in [Co] (See also Theorems 9.31 and 9.40 in [Ch]).

Theorem 6.1 (Volume convergence). Suppose that (5.1) and (5.2) are satisfied, and

$$
d_{G H}(B(x, R), \underline{B}(0, R)) \leq \epsilon,
$$

for some $R \leq 1$ and sufficiently small $\epsilon>0$. Then for some $\Psi=\Psi(\lambda, K, \epsilon \mid n, R, \alpha, \rho)$ we have

$$
\operatorname{Vol}(B(x, R)) \geq(1-\Psi) \operatorname{Vol}(\underline{B}(0, R)) .
$$

Here $\underline{B}(0, R)$ is the ball in $\mathbb{R}^{n}$.

The above theorem implies the volume continuity under Gromov-Hausdorff convergence. 
Theorem 6.2 (Volume continuity). For a sequence of Riemannian manifolds $\left\{\left(\mathbf{M}_{i}, g_{i}\right)\right\}$, assume that

$$
\operatorname{Ric}_{\mathbf{M}_{i}}+\frac{1}{2} \mathcal{L}_{V_{i}} g_{\mathbf{M}_{i}} \geq-\lambda g_{\mathbf{M}_{i}},\left|V_{i}\right|\left(y_{i}\right) \leq \frac{K}{d_{i}^{\alpha}\left(y_{i}, O_{i}\right)}, \text { and } \operatorname{Vol}\left(B\left(y_{i}, 1\right)\right) \geq \rho,
$$

where $O_{i}$ is a fixed point in $\mathbf{M}_{i}$ and $y_{i}$ is an arbitrary point in $\mathbf{M}_{i}$.

Let $(\mathbf{M}, g)$ be a Riemannian manifold. If $\left(\mathbf{M}_{i}, d_{i}, x_{i}\right) \stackrel{d_{G H}}{\longrightarrow}(\mathbf{M}, d, x)$ in pointed GromovHausdorff topology, then for all $r<\infty$,

$$
\operatorname{Vol}\left(B\left(x_{i}, r\right)\right)=\operatorname{Vol}(B(x, r)) .
$$

Next, we show a cone rigidity theorem in the spirit of [ChCo1. Although our proof follows the idea there, certain modifications and extensions in the choice of the axillary functions are needed.

For a metric space $(Z, d)$, denote by $(C(Z), \hat{d})$ the metric cone on $Z$. That is, $C(Z)=$ $(0, \infty) \times Z$ with metric:

$$
\hat{d}\left(\left(r_{1}, z_{1}\right),\left(r_{2}, z_{2}\right)\right)=\left\{\begin{array}{cl}
r_{1}^{2}+r_{2}^{2}-2 r_{1} r_{2} \cos \left(d\left(z_{1}, z_{2}\right)\right), & \text { if } d\left(z_{1}, z_{2}\right) \leq \pi \\
r_{1}+r_{2}, & \text { if } d\left(z_{1}, z_{2}\right)>\pi
\end{array}\right.
$$

In the following, unless otherwise specified, $C_{i}$ will denote constants depending on $n, \lambda, K, \alpha$, and $\rho$.

Theorem 6.3 (Cone rigidity). Suppose that (5.1) and (5.2) are satisfied. There exists a $r_{0}=r_{0}(n, \lambda, K, \alpha, \rho)$ such that if for $R \leq r_{0}$ and $\delta>0$,

$$
(1-\delta) \operatorname{Vol}(B(x, R)) \leq \frac{R}{n} \operatorname{Vol}(\partial B(x, R)),
$$

then for some $\Psi=\Psi(\lambda, K, \delta \mid n, R, \alpha, \rho)$, we have

$$
d_{G H}\left(B(x, R), C_{0, R}(Z)\right) \leq \Psi
$$

where $Z$ is a length space with

$$
\operatorname{diam}(Z) \leq \pi+\Psi
$$

Proof. Step 1. We show that the metric is close to the Hessian of a function in average sense.

First, we derive bounds on $f$. In [ChCo1] (see also [Ch]), the lower bound of $f$ was obtained by constructing certain comparison function $\underline{L}_{R}$ and using the maximum principle on $f-\underline{L}_{3 R}\left(r_{y}\right)$, where $y$ is an arbitrary point in $\mathbf{M}$ with $d(y, x)=2 R$ and $r_{y}$ is the distance function from $y$. The same method can be applied to drive the lower bound of $f$ in this case as well. However, here we will use Dirichlet Green's function for simplicity.

For any open subset $\Omega$ in $\mathbf{M}$, denote by $\Gamma_{\Omega}(z, y)$ the Dirichlet Green's function in $\Omega$. That is,

$$
\Delta_{x} \Gamma_{\Omega}(z, y)=-\delta_{y} ; \Gamma_{\Omega}(z, y)=\Gamma_{\Omega}(y, z) ;\left.\Gamma_{\Omega}(\cdot, y)\right|_{\partial \Omega}=0 .
$$

Given $x \in \mathbf{M}$, let $f$ be the smooth solution of the following Dirichlet problem:

$$
\Delta f=1 \text { in } B(x, R) ;\left.f\right|_{\partial B(x, R)}=\frac{R^{2}}{2 n} .
$$


By using the Green's function, we can write $f$ as

$$
f(z)=\frac{R^{2}}{2 n}-\int_{B(x, R)} \Gamma_{B(x, R)}(z, y) d g(y) .
$$

By the upper bound of the heat kernel in Theorem 4.4, we have

$$
\begin{aligned}
\Gamma_{B(x, R)}(z, y) & \leq \int_{0}^{1} G(z, t ; y, 0) d t+\int_{1}^{\infty} C e^{-c t} d t \\
& \leq \int_{0}^{1} C_{3} t^{-\frac{n}{2}} e^{-\frac{d^{2}(z, y)}{C_{4} t}} d t+C \leq C_{5} d^{-(n-2)}(z, y) .
\end{aligned}
$$

Here we have used the large time exponential decay of the Dirichlet heat kernel on a ball. Note that this decay can be obtained by proving that the $L^{2}$ norm decays exponentially first. This needs the help of the Poincaré inequality on a ball, which is true due the the heat kernel bound and gradient estimate. Then one can apply the mean value inequality. Therefore, (6.4) and (6.5) imply

$$
f(z) \geq \frac{R^{2}}{2 n}-\int_{B(x, R)} C_{5} d^{-(n-2)}(z, y) d g(y) \geq \frac{R^{2}}{2 n}-C(n) e^{C(\alpha) K R^{1-\alpha}+4 \lambda R^{2}} R^{2} .
$$

This gives a lower bound of $f$.

For an upper bound of $f$, let $r_{x}$ be the distance function from $x$. Then

$$
\Delta f-\Delta \frac{r_{x}^{2}}{2 n}=1-\frac{r_{x}}{n} \Delta r_{x}-\frac{1}{n} \geq-\frac{r_{x}}{n} \psi
$$

where $\psi=\left(\Delta r_{x}-\frac{n-1}{r_{x}}\right)_{+}$. From Proposition 2.1, one has

$$
\psi \leq \frac{\lambda}{3} r_{x}+|V|+\frac{C(\alpha) K}{r_{x}^{\alpha}}
$$

Thus,

$$
r_{x} \psi \leq r_{x}\left(\frac{\lambda}{3} r_{x}+|V|+\frac{C(\alpha) K}{r_{x}^{\alpha}}\right) \leq \lambda R^{2}+R|V|+C(\alpha) K R^{1-\alpha},
$$

and (6.7) becomes

$$
\Delta f-\Delta \frac{r_{x}^{2}}{2 n} \geq-C(n)\left(\lambda R^{2}+C(\alpha) K R^{1-\alpha}\right)-C(n) R|V| .
$$

According to Theorem 4.5, we get

$$
\begin{aligned}
f(z) & \leq \frac{r_{x}^{2}(z)}{2 n}+C(n) R^{2}\left(\lambda R^{2}+C(\alpha) K R^{1-\alpha}\right)+C(n) R^{3}\|V\|_{q, B(x, R)}^{*} \\
& \leq \frac{r_{x}^{2}(z)}{2 n}+C(n, \lambda, K, \alpha, \rho)\left(\lambda R^{4}+K R^{3-\alpha}\right) .
\end{aligned}
$$

Next, we show that $f$ and $\frac{r_{x}^{2}}{2 n}$ are close. By the volume condition (6.1) in the theorem,

$$
\begin{aligned}
\int_{B(x, R)} \Delta f-\Delta \frac{r_{x}^{2}}{2 n} & =\operatorname{Vol}(B(x, R))-\int_{\partial B(x, R)}<\nabla \frac{r_{x}^{2}}{2 n}, \nabla r_{x}>d S \\
& =\operatorname{Vol}(B(x, R))-\frac{R}{n} \operatorname{Vol}(\partial B(x, R)) \\
& \leq \delta \operatorname{Vol}(B(x, R)) .
\end{aligned}
$$


From (6.8), we can also get the lower bound

$$
\begin{aligned}
\oint_{B(x, R)} \Delta f-\Delta \frac{r_{x}^{2}}{2 n} & \geq-\oint_{B(x, R)} C(n)\left(\lambda R^{2}+C(\alpha) K R^{1-\alpha}\right)+C(n) R|V| \\
& \geq-C(n, \lambda, K, \alpha, \rho)\left(\lambda R^{2}+K R^{1-\alpha}\right) .
\end{aligned}
$$

Therefore, we have

$$
-C(n, \lambda, K, \alpha, \rho)\left(\lambda R^{2}+K R^{1-\alpha}\right) \leq \oint_{B(x, R)} \Delta f-\Delta \frac{r_{x}^{2}}{2 n} \leq \delta .
$$

Also, in (6.9) , setting $A:=C(n, \lambda, K, \alpha, \rho)\left(\lambda R^{4}+K R^{3-\alpha}\right)$, the constant on the right hand side, yields

$$
\frac{r_{x}^{2}}{2 n}-f+A \geq 0
$$

Combining the inequality above and (6.6), (6.7), 6.10) and Lemma 2.4, we have

$$
\begin{aligned}
0 & \leq \oint_{B(x, R)}\left[\Delta\left(f-\frac{r_{x}^{2}}{2 n}\right)+\frac{r_{x}}{n} \psi\right]\left[\frac{r_{x}^{2}}{2 n}-f+A\right] \\
& =\oint_{B(x, R)} A \Delta\left(f-\frac{r_{x}^{2}}{2 n}\right)+\left(\frac{r_{x}^{2}}{2 n}-f\right) \Delta\left(f-\frac{r_{x}^{2}}{2 n}\right)+\frac{r_{x}}{n} \psi\left[\frac{r_{x}^{2}}{2 n}-f+A\right] \\
& \leq \delta A-\oint_{B(x, R)}\left|\nabla\left(f-\frac{r_{x}^{2}}{2 n}\right)\right|^{2}+\frac{1}{n}\left[C(n) e^{C(\alpha) K R^{1-\alpha}+4 \lambda R^{2}} R^{2}+A\right]\left(\lambda R^{2}+C(\alpha) K R^{1-\alpha}\right) .
\end{aligned}
$$

It follows that

$$
\begin{aligned}
\oint_{B(x, R)}\left|\nabla\left(f-\frac{r_{x}^{2}}{2 n}\right)\right|^{2} & \leq \delta A+\frac{1}{n}\left[C(n) e^{C(\alpha) K R^{1-\alpha}+4 \lambda R^{2}} R^{2}+A\right]\left(\lambda R^{2}+C(\alpha) K R^{1-\alpha}\right) \\
& =\Psi(\delta, \lambda, K \mid n, R, \alpha, \rho)
\end{aligned}
$$

Therefore, by the Poincaré inequality, we have

$$
\oint_{B(x, R)}\left(\frac{r_{x}^{2}}{2 n}-f\right)^{2} \leq \Psi(\delta, \lambda, K \mid n, R, \alpha, \rho) .
$$

From (6.13), volume comparison theorem (2.2), the gradient estimate (4.1), and the bounds of $f((\underline{6.6})$ and $(\underline{6.9}))$, it is not hard to see that

$$
\left|\frac{r_{x}^{2}}{2 n}-f\right| \leq \Psi_{1}(\delta, \lambda, K \mid n, R, \alpha, \rho), \text { on } B\left(x,\left(1-\Psi_{1}\right) R\right),
$$

where $\Psi_{1}=\Psi^{1 /(n+1)}$.

Finally, we show the closeness of $\nabla^{2} f$ and the metric $g$ in the average sense. Note that, by Theorems 4.1 and 4.5 , we have $|\nabla f| \leq C$ in $B\left(x,\left(1-\Psi_{1}\right) R\right.$ ). This together with (6.14) and (6.12) gives

$$
\left.\oint_{B\left(x,\left(1-\Psi_{1}\right) R\right)}|| \nabla f\right|^{2}-\frac{2 f}{n} \mid \leq \Psi_{1}
$$


Now, let $\phi$ be the cut-off function as in Lemma 4.6 with $\left.\phi\right|_{B\left(x,\left(1-\Psi_{1}\right) R\right)}=1$. From (6.15) and Bochner's formula, we have

$$
\begin{aligned}
C R^{-2} \Psi_{1} & \geq \frac{1}{2} \oint_{B(x, R)} \Delta \phi \cdot\left(|\nabla f|^{2}-\frac{2 f}{n}\right) \\
& \geq \frac{1}{2} \oint_{B(x, R)} \phi \cdot \Delta\left(|\nabla f|^{2}-\frac{2 f}{n}\right) \\
& =\oint_{B(x, R)} \phi\left(\left|\nabla^{2} f\right|^{2}+\operatorname{Ric}(\nabla f, \nabla f)-\frac{1}{n}\right) \\
& \geq \oint_{B(x, R)} \phi\left(\left|\nabla^{2} f\right|^{2}-\frac{1}{n}-\lambda|\nabla f|^{2}-\nabla_{i} V_{j} f_{i} f_{j}\right) .
\end{aligned}
$$

Using Lemma 2.4 and the boundedness of $|\nabla f|$, we obtain

$$
\begin{aligned}
-\oint_{B(x, R)} \phi \nabla_{i} V_{j} f_{i} f_{j} & =\oint_{B(x, R)} \phi_{i} V_{j} f_{i} f_{j}+\phi V_{j} f_{j}+\phi V_{j} f_{i} f_{i j} \\
& \geq-C R^{-1} \oint_{B(x, R)}|V|-\left(\oint_{B(x, R)} \phi|V|^{2}|\nabla f|^{2}\right)^{1 / 2} \cdot\left(\oint_{B(x, R)} \phi\left|\nabla^{2} f\right|^{2}\right)^{1 / 2} \\
& \geq-C R^{-1-\alpha} K-\frac{1}{4} K^{-1 / 2} \oint_{B(x, R)} \phi|V|^{2}|\nabla f|^{2}-K^{1 / 2} \oint_{B(x, R)} \phi\left|\nabla^{2} f\right|^{2} \\
& \geq-C R^{-1-\alpha} K-C R^{-2 \alpha} K^{3 / 2}-K^{1 / 2} \oint_{B(x, R)} \phi\left|\nabla^{2} f\right|^{2} .
\end{aligned}
$$

It then follows from (6.16) and (6.17) that

$$
\Psi_{1} \geq-C\left(\lambda R^{2}+R^{1-\alpha} K+R^{2-2 \alpha} K^{3 / 2}\right)+C R^{2} \oint_{B(x, R)} \phi\left(1-K^{1 / 2}\right)\left[\left|\nabla^{2} f\right|^{2}-\frac{1}{n}\right]-K^{1 / 2} \frac{1}{n} .
$$

That is

$$
\begin{aligned}
\oint_{B(x, R)} \phi\left[\left|\nabla^{2} f\right|^{2}-\frac{1}{n}\right] & \leq\left(1-K^{1 / 2}\right)^{-1}\left[\Psi_{1}+C\left(\lambda R^{2}+R^{1-\alpha} K+R^{2-2 \alpha} K^{3 / 2}+K^{1 / 2} R^{2}\right)\right] \\
& =\Psi_{2} .
\end{aligned}
$$

Therefore,

$$
\oint_{B\left(x,\left(1-\Psi_{1}\right) R\right)}\left|\nabla^{2} f-\frac{1}{n} g_{i j}\right|^{2} \leq \oint_{B(x, R)} \phi\left[\left|\nabla^{2} f\right|^{2}-\frac{1}{n}\right] \leq \Psi_{2} .
$$

Step 2. Now we may follow the argument in section 3 and 4 in [ChCo1] (see also Theorem 9.45 of [Ch]) to show (6.2) and (6.3).

Roughly speaking, for any points $y, z, w \in B\left(x,\left(1-\Psi_{1}\right) R\right)$, with $r_{x}(y)=r_{x}(z)=a$ and $z$ being the point on $r_{x}^{-1}(a)$ closest to $w$, by using the segment inequality (5.4), (6.15) and (6.18), one can find $y^{*}, z^{*}, w^{*}$ close to $y, z, w$, respectively, and

$$
\int_{r_{x}\left(z^{*}\right)}^{r_{x}\left(z^{*}\right)+d\left(z^{*}, w^{*}\right)}\left|\nabla^{2} f\right|\left(\gamma_{s}(t)\right) d t d s \leq \Psi
$$


and

$$
\left.\int_{r_{x}\left(z^{*}\right)}^{r_{x}\left(z^{*}\right)+d\left(z^{*}, w^{*}\right)}|| \nabla f\right|^{2}-\frac{2 f}{n} \mid(\sigma(s)) d s \leq \Psi .
$$

Here $\sigma(s), r_{x}\left(z^{*}\right) \leq s \leq r_{x}\left(z^{*}\right)+d\left(z^{*}, w^{*}\right)$, is the minimal geodesic from $z^{*}$ to $w^{*}$, and $\gamma_{s}:[0, l(s)] \rightarrow \mathbf{M}$ the minimal geodesic from $x^{*}$ to $\sigma(s)$.

From (6.19), (6.20) and the first variation of arc length, it then follows that $d\left(x^{*}, w^{*}\right)$ is close to the distance between them when they are considered to be two points in a metric cone on $r_{x}^{-1}(a)$.

As pointed out in [Ch], when applying Theorem 6.3, it is often more convenient to verify the following condition instead of (6.1).

Lemma 6.4. If

$$
\left(1-\delta^{\prime}\right) \frac{\operatorname{Vol}\left(B\left(x, \frac{1}{2} R\right)\right)}{\operatorname{Vol}\left(\underline{B}\left(0, \frac{1}{2} R\right)\right)} \leq \frac{\operatorname{Vol}(B(x, R))}{\operatorname{Vol}(\underline{B}(0, R))},
$$

then for any $0<\eta \leq \frac{1}{2}$ and some $\Psi=\Psi\left(\delta^{\prime}, \lambda, K \mid n, \alpha, \rho\right)$, we have

$$
(1-\Psi) \frac{\operatorname{Vol}(B(x,(1-\eta) R))}{\operatorname{Vol}(\underline{B}(0,(1-\eta) R))} \leq \frac{\operatorname{Vol}(\partial B(x,(1-\eta) R))}{\operatorname{Vol}(\partial \underline{B}(0,(1-\eta) R))},
$$

i.e.,

$$
(1-\Psi) \operatorname{Vol}(B(x,(1-\eta) R)) \leq \frac{R}{n} \operatorname{Vol}(\partial B(x,(1-\eta) R)) .
$$

Proof. See appendix II.

From Lemma 6.4 and Theorem 6.3, one can follow a similar argument as in Theorem 9.69 in $\mathrm{Ch}$ to show that

Theorem 6.5. Suppose that (5.1) and (5.2) are satisfied. There exists an $r_{0}=r_{0}(n, \lambda, K, \alpha, \rho)$ such that if

$$
\operatorname{Vol}(B(x, R)) \geq(1-\epsilon) \operatorname{Vol}(\underline{B}(0, R)),
$$

for some $R \leq r_{0}$ and sufficiently small $\epsilon>0$, then

$$
d_{G H}(B(x, R), \underline{B}(0, R)) \leq \Psi(\lambda, K, \epsilon \mid n, R, \alpha, \rho) .
$$

Theorem 6.3 also implies that the tangent cones of a Gromov-Hausdorff limit are metric cones as shown in Theorem 5.2 in ChCo2.

Theorem 6.6 (Tangent cones are metric cones). Let $\left(\mathbf{M}_{i}, g_{i}\right)$ be a sequence of Riemannian manifolds satisfying (5.1) and (5.2) for some $\lambda, K, \alpha$, and $\rho$. Suppose that $\left(\mathbf{M}_{i}, d_{i}\right) \stackrel{d_{G H}}{\longrightarrow}$ $(Y, d)$ in Gromov-Hausdorff topology. Then any tangent cone of $Y$ is a metric cone.

Finally, following the proof of Theorem 6.1 in ChCo2 closely, we deduce

Theorem 6.7 (Size estimate of the singular set). Let $\left(\mathbf{M}_{i}, g_{i}\right)$ be a sequence of Riemannian manifolds satisfying (5.1) and (15.2) for some $\lambda, K, \alpha$, and $\rho$. Suppose that $\left(\mathbf{M}_{i}, d_{i}\right) \stackrel{d_{G H}}{\longrightarrow}$ $(Y, d)$ in Gromov-Hausdorff topology. Then $\operatorname{dim} S(Y) \leq n-2$. Here, $S(Y)$ is the singular set of $Y$ and dim stands for the Hausdorff dimension. 
Recall that $S(Y)$ is the set of points in $Y$ such that a tangent cone at $y$ is not isometric to a Euclidean space.

To prove the theorem, one just needs to show that no tangent cone is isometric to the half Euclidean space. We mention that a small modification is needed in the proof. Namely, as pointed out in the proof of Theorem 6.1 [ChCo2], one can replace the usage of Perelman's theorem in [Pe] by using Theorem A.1.8 in [ChCo2]. The conclusion of this later theorem continues to hold in our situation since it follows from the conclusions of Theorem A.1.1 in and Theorem A.1.5 in ChCo2]. But Theorem A.1.1 in [ChCo2 is a version of Reifenberg's theorem [Re] which is a result for more general metric spaces. So it is still valid here. The conclusion of Theorem A.1.5 is still true here due to Theorem 6.5 above. So the map $\hat{f}$ in the proof of Theorem 6.1 ChCo2 is an imbedding. Therefore it is mod 2 degree is 1 . But from p437 in [ChCo2, its mod 2 degree is also 0. This contradiction finishes the proof.

Remark 6.8. In the case of Bakry-Émery Ricci curvature condition, the conclusions of the theorems in this section continue to hold under conditions (2.43) and (2.44). Namely, one only needs the potential function $L$ to be Hölder continuous and its gradient in certain $L^{p}$ space. In this case it is not clear whether the segment inequality5.1 still hold. However, a weaker form of it is available from the work [TZ2], which is sufficient for the proof of the main results.

Remark 6.9. With a little more effort, along the lines of Section 3 in [PeWe1], one can also prove some compactness and finiteness results of topological types under conditions (5.1) and (5.2) and extra integral assumption on the curvature tensor. Also, with two sided bounds on the Ricci curvature with two vector fields $U$ and $V$, namely,

$\frac{1}{2} \mathcal{L}_{U} g+\lambda g \geq \operatorname{Ric} \geq-\frac{1}{2} \mathcal{L}_{V} g-\lambda g ; \quad|U|+|V|(y) \leq \frac{K}{d(y, O)^{\alpha}}, \alpha \in[0,1) ; \quad \operatorname{Vol}(B(x, 1)) \geq \rho$,

one can also prove that $S(Y)$ is a closed set using the technique in ChCo2] and the results here.

\section{Appendix I}

In this section, we give a proof of Theorem 4.1, namely

Theorem 7.1. Assume that

$$
\operatorname{Ric}+\frac{1}{2} \mathcal{L}_{V} g \geq-\lambda g, \quad|V|(y) \leq \frac{K}{d(y, O)^{\alpha}}, \quad \alpha \in[0,1), \quad \operatorname{Vol}(B(x, 1)) \geq \rho .
$$

Then there exists a positive constant $r_{0}=r_{0}(n, \lambda, K, \alpha, \rho) \leq 1$ such that, for any $x \in \mathbf{M}$, $0<r \leq r_{0}$ and smooth functions $u$ and $f$ satisfying the equation

$$
\Delta u=f, \text { in } B(x, r) \text {, }
$$

we have

$$
\sup _{B\left(x, \frac{1}{2} r\right)}|\nabla u|^{2} \leq C(n, \lambda, K, \alpha, \rho) r^{-2}\left[\left(\|u\|_{2, B(x, r)}^{*}\right)^{2}+\left(\|f\|_{2 q, B(x, r)}^{*}\right)^{2}\right],
$$

for any $q>n / 2$. Moreover

$$
\sup _{B\left(x, \frac{1}{2} r\right)} u^{2} \leq C(n, \lambda, K, \alpha, \rho)\left[\left(\|u\|_{2, B(x, r)}^{*}\right)^{2}+\left(\|f\|_{q, B(x, r)}^{*}\right)^{2}\right] .
$$


In particular if $\alpha=0$, then the conclusions hold without the non-collapsing condition.

Proof. Let

$$
v=|\nabla u|^{2}+\left\|f^{2}\right\|_{q, B(x, r)}^{*}
$$

Then

$$
\begin{aligned}
\Delta v & =2\left|\nabla^{2} u\right|^{2}+2<\nabla \Delta u, \nabla u>+2 \operatorname{Ric}(\nabla u, \nabla u) \\
& \geq 2 u_{i} f_{i}-2 \lambda v-\left(\mathcal{L}_{V} g\right)_{i j} u_{i} u_{j} .
\end{aligned}
$$

For any $p>0$,

$$
\begin{aligned}
\Delta v^{p} & =p v^{p-1} \Delta v+p(p-1) v^{p-2}|\nabla v|^{2} \\
& \geq 2 p v^{p-1} u_{i} f_{i}-2 \lambda p v^{p}-p v^{p-1}\left(\mathcal{L}_{V} g\right)_{i j} u_{i} u_{j}+\frac{p-1}{p} v^{-p}\left|\nabla v^{p}\right|^{2} .
\end{aligned}
$$

Let $B=B(x, r)$ and $p \geq 1$, then it follows from (7.2) that

$$
\begin{aligned}
\int_{B}\left|\nabla\left(\eta v^{p}\right)\right|^{2} & =\int_{B}\left|\eta \nabla v^{p}+v^{p} \nabla \eta\right|^{2} \\
& =\int_{B} \eta^{2}\left|\nabla v^{p}\right|^{2}+v^{2 p}|\nabla \eta|^{2}+2 \eta v^{p}<\nabla v^{p}, \nabla \eta> \\
& =\int_{B} v^{2 p}|\nabla \eta|^{2}-\eta^{2} v^{p} \Delta v^{p} \\
& \leq \int_{B} v^{2 p}|\nabla \eta|^{2}-2 p \eta^{2} v^{2 p-1} u_{i} f_{i}+2 \lambda p \eta^{2} v^{2 p}+p \eta^{2} v^{2 p-1}\left(\mathcal{L}_{V} g\right)_{i j} u_{i} u_{j} .
\end{aligned}
$$

Moreover, since

$$
\begin{aligned}
& \int_{B} \eta^{2} v^{2 p-1}\left(\mathcal{L}_{V} g\right)_{i j} u_{i} u_{j} \\
= & \int_{B} \eta^{2} v^{2 p-1} \nabla_{j} V_{i} u_{i} u_{j} \\
= & -\int_{B} 2 \eta v^{2 p-1} \eta_{j} V_{i} u_{i} u_{j}+(2 p-1) \eta^{2} v^{2 p-2} v_{j} V_{i} u_{i} u_{j}+\eta^{2} v^{2 p-1} V_{i} u_{i j} u_{j}+\eta^{2} v^{2 p-1} f V_{i} u_{i} \\
\leq & \int_{B} v^{2 p}|\nabla \eta|^{2}+\eta^{2} v^{2 p-2}|V|^{2}|\nabla u|^{4}-\frac{2 p-1}{p} \eta v^{p-1} V_{i} u_{i} u_{j}\left[\left(\eta v^{p}\right)_{j}-v^{p} \eta_{j}\right]-\frac{1}{2} \eta^{2} v^{2 p-1} V_{i} v_{i} \\
& +\frac{1}{2} \eta^{2} v^{2 p-2} f^{2}|\nabla u|^{2}+\frac{1}{2} \eta^{2} v^{2 p}|V|^{2} \\
\leq & \int_{B} v^{2 p}|\nabla \eta|^{2}+\frac{3}{2} \eta^{2} v^{2 p}|V|^{2}-\frac{2 p-1}{p} \eta v^{p-1} V_{i} u_{i} u_{j}\left[\left(\eta v^{p}\right)_{j}-v^{p} \eta_{j}\right] \\
& \quad-\frac{1}{2 p} \eta^{2} v^{p} V_{i}\left[\left(\eta v^{p}\right)_{i}-v^{p} \eta_{i}\right]+\frac{1}{2} \eta^{2} v^{2 p-2} f^{2}|\nabla u|^{2}
\end{aligned}
$$




$$
\begin{aligned}
& \leq \int_{B} v^{2 p}|\nabla \eta|^{2}+\frac{3}{2} \eta^{2} v^{2 p}|V|^{2}+\frac{1}{4 p}\left|\nabla\left(\eta v^{p}\right)\right|^{2}+\frac{(2 p-1)^{2}}{p} \eta^{2} v^{2 p}|V|^{2}+\frac{2 p-1}{2 p} v^{2 p}|\nabla \eta|^{2} \\
& \quad+\frac{2 p-1}{2 p} \eta^{2} v^{2 p}|V|^{2}+\frac{1}{4 p}\left|\nabla\left(\eta v^{p}\right)\right|^{2}+\frac{1}{4 p} \eta^{2} v^{2 p}|V|^{2}+\frac{1}{4 p} \eta^{2} v^{2 p}|V|^{2} \\
& \quad+\frac{1}{4 p} v^{2 p}|\nabla \eta|^{2}+\frac{1}{2} \eta^{2} v^{2 p-1} f^{2} \\
& =\int_{B} \frac{8 p-1}{4 p} v^{2 p}|\nabla \eta|^{2}+\frac{2(2 p-1)^{2}+5 p}{2 p} \eta^{2} v^{2 p}|V|^{2}+\frac{1}{2 p}\left|\nabla\left(\eta v^{p}\right)\right|^{2}+\frac{1}{2} \eta^{2} v^{2 p-1} f^{2},
\end{aligned}
$$

we can rewrite (7.3) as

$$
\begin{gathered}
\int_{B}\left|\nabla\left(\eta v^{p}\right)\right|^{2} \leq \int_{B} 2 v^{2 p}|\nabla \eta|^{2}-4 p \eta^{2} v^{2 p-1} u_{i} f_{i}+4 \lambda p \eta^{2} v^{2 p}+\frac{8 p-1}{2} v^{2 p}|\nabla \eta|^{2} \\
+\left(2(2 p-1)^{2}+5 p\right) \eta^{2} v^{2 p}|V|^{2}+p \eta^{2} v^{2 p-1} f^{2}
\end{gathered}
$$

Notice that

$$
\begin{aligned}
& -\int_{B} \eta^{2} v^{2 p-1} u_{i} f_{i} \\
= & \int_{B} \eta^{2} v^{2 p-1} f^{2}+2 \eta v^{2 p-1} f \nabla_{i} u \nabla_{i} \eta+(2 p-1) \eta^{2} v^{2 p-2} f \nabla_{i} u \nabla_{i} v \\
= & \int_{B} \eta^{2} v^{2 p-1} f^{2}+2 \eta v^{2 p-1} f \nabla_{i} u \nabla_{i} \eta+\frac{2 p-1}{p} \eta v^{p-1} f \nabla_{i} u\left(\nabla_{i}\left(\eta v^{p}\right)-v^{p} \nabla_{i} \eta\right) \\
= & \int_{B} \eta^{2} v^{2 p-1} f^{2}+\frac{1}{p} \eta v^{2 p-1} f \nabla_{i} u \nabla_{i} \eta+\frac{2 p-1}{p} \eta v^{p-1} f \nabla_{i} u \nabla_{i}\left(\eta v^{p}\right) \\
\leq & \int_{B} \eta^{2} v^{2 p-1} f^{2}+\frac{1}{2 p} \eta^{2} v^{2 p-2} f^{2}|\nabla u|^{2}+\frac{1}{2 p} v^{2 p}|\nabla \eta|^{2}+\frac{1}{8 p}\left|\nabla\left(\eta v^{p}\right)\right|^{2}+\frac{2(2 p-1)^{2}}{p} \eta^{2} v^{2 p-2} f^{2}|\nabla u|^{2} \\
\leq & \int_{B} \frac{4(2 p-1)^{2}+1}{2 p} \eta^{2} v^{2 p-1} f^{2}+\frac{1}{2 p} v^{2 p}|\nabla \eta|^{2}+\frac{1}{8 p}\left|\nabla\left(\eta v^{p}\right)\right|^{2} .
\end{aligned}
$$

Thus, it follows that

$$
\begin{gathered}
\int_{B}\left|\nabla\left(\eta v^{p}\right)\right|^{2} \leq \int_{B} 4 v^{2 p}|\nabla \eta|^{2}+8 \lambda p \eta^{2} v^{2 p}+(8 p-1) v^{2 p}|\nabla \eta|^{2}+\left(4(2 p-1)^{2}+10 p\right) \eta^{2} v^{2 p}|V|^{2} \\
+2 p \eta^{2} v^{2 p-1} f^{2}+\left(16(2 p-1)^{2}+4\right) \eta^{2} v^{2 p-1} f^{2}+4 v^{2 p}|\nabla \eta|^{2}
\end{gathered}
$$

Assume that $r_{i}=\left(\frac{1}{2}+\frac{1}{2^{i+2}}\right) r, i=0,1,2, \cdots$. Construct cut-off function $\phi_{i}(s)$ such that

$$
\operatorname{supp} \phi_{i} \subseteq\left[0, r_{i}\right], \quad \phi_{i}=1 \text { on }\left[0, r_{i+1}\right], \text { and }-\frac{52^{i}}{r} \leq \phi_{i}^{\prime} \leq 0 ;
$$

Let $\eta_{i}(y)=\phi_{i}(d(y, x))$. Then (7.5) implies

$$
\oint_{B\left(x, r_{i}\right)}\left|\nabla\left(\eta_{i} v^{p}\right)\right|^{2} \leq \oint_{B\left(x, r_{i}\right)} 8 \lambda p \eta_{i}^{2} v^{2 p}+16 p v^{2 p}\left|\nabla \eta_{i}\right|^{2}+30 p^{2} \eta_{i}^{2} v^{2 p}|V|^{2}+70 p^{2} \eta_{i}^{2} v^{2 p-1} f^{2} .
$$


On the other hand, since $\frac{r}{2} \leq r_{i} \leq \frac{3}{4} r$, by the volume comparison theorem

$$
\begin{aligned}
& p^{2} \oint_{B\left(x, r_{i}\right)} \eta_{i}^{2} v^{2 p-1} f^{2} \\
\leq & \frac{p^{2}}{\left\|f^{2}\right\|_{q, B(x, r)}^{*}} \oint_{B\left(x, r_{i}\right)} \eta_{i}^{2} v^{2 p} f^{2} \\
\leq & C(n, \lambda, K, \alpha, \rho) p^{2}\left(\oint_{B\left(x, r_{i}\right)}\left(\eta_{i} v^{p}\right)^{\frac{2 q}{q-1}}\right)^{\frac{q-1}{q}} \\
\leq & C(n, \lambda, K, \alpha, \rho) p^{2}\left(\oint_{B\left(x, r_{i}\right)}\left(\eta_{i} v^{p}\right)^{a \cdot \frac{2 q}{q-1} \cdot b}\right)^{\frac{q-1}{q b}} \cdot\left(\oint_{B\left(x, r_{i}\right)}\left(\eta_{i} v^{p}\right)^{(1-a) \cdot \frac{2 q}{q-1} \cdot \frac{b}{b-1}}\right)^{\frac{(q-1)(b-1)}{q b}} \\
\leq & \epsilon\left(\oint_{B\left(x, r_{i}\right)}\left(\eta_{i} v^{p}\right)^{a \cdot \frac{2 q}{q-1} \cdot b}\right)^{\frac{q-1}{q b a}}+\epsilon^{-\frac{a}{1-a}} C^{\frac{1}{1-a}} p^{\frac{2}{(1-a)}}\left(\oint_{B\left(x, r_{i}\right)}\left(\eta_{i} v^{p}\right)^{(1-a) \cdot \frac{2 q}{q-1} \cdot \frac{b}{b-1}}\right)^{\frac{(q-1)(b-1)}{q b(1-a)}} .
\end{aligned}
$$

Here in the last step, we have used Young's inequality

$$
x y \leq \epsilon x^{\gamma}+\epsilon^{-\frac{\gamma^{*}}{\gamma}} y^{\gamma^{*}}, \forall x, y>0, \gamma>1, \frac{1}{\gamma}+\frac{1}{\gamma^{*}}=1,
$$

for $\gamma=\frac{1}{a}$.

Since $q>\frac{n}{2}$, if we choose $a=\frac{n}{2 q}$, and $b=\frac{2 q-2}{n-2}$, it follows from (17.7) that

$$
p^{2} \oint_{B\left(x, r_{i}\right)} \eta_{i}^{2} v^{2 p-1} f^{2} \leq \epsilon\left(\oint_{B\left(x, r_{i}\right)}\left(\eta_{i} v^{p}\right)^{\frac{2 n}{n-2}}\right)^{\frac{n-2}{n}}+\epsilon^{-\frac{a}{1-a}} C^{\frac{2 q}{2 q-n}} p^{\frac{4 q}{2 q-n}} \oint_{B\left(x, r_{i}\right)} \eta_{i}^{2} v^{2 p} .
$$

Hence, (7.6) becomes

$$
\begin{aligned}
\oint_{B\left(x, r_{i}\right)}\left|\nabla\left(\eta_{i} v^{p}\right)\right|^{2} \leq & \oint_{B\left(x, r_{i}\right)} 8 \lambda p \eta_{i}^{2} v^{2 p}+16 p v^{2 p}\left|\nabla \eta_{i}\right|^{2}+30 p^{2} \eta_{i}^{2} v^{2 p}|V|^{2} \\
& +70 \epsilon\left(\oint_{B\left(x, r_{i}\right)}\left(\eta_{i} v^{p}\right)^{\frac{2 n}{n-2}}\right)^{\frac{n-2}{n}}+70 \epsilon^{-\frac{a}{1-a}} C^{\frac{2 q}{2 q-n}} p^{\frac{4 q}{2 q-n}} \oint_{B\left(x, r_{i}\right)} \eta_{i}^{2} v^{2 p} .
\end{aligned}
$$

If $q \in\left(\frac{n}{2}, \frac{n}{2 \alpha}\right)$, then

$$
\begin{aligned}
& 30 p^{2} \oint_{B\left(x, r_{i}\right)} \eta_{i}^{2} v^{2 p}|V|^{2} \\
\leq & 30 p^{2}\left(\oint_{B\left(x, r_{i}\right)}\left(\eta_{i} v^{p}\right)^{\frac{2 q}{q-1}}\right)^{\frac{q-1}{q}} \cdot\left(\oint_{B\left(x, r_{i}\right)}|V|^{2 q}\right)^{1 / q} \\
\leq & p^{2} C(n, \lambda, K, \alpha, \rho) r_{i}^{-2 \alpha}\left(\oint_{B\left(x, r_{i}\right)}\left(\eta_{i} v^{p}\right)^{\frac{2 q}{q-1}}\right)^{\frac{q-1}{q}} \\
\leq & \epsilon r_{i}^{-2 \alpha}\left(\oint_{B\left(x, r_{i}\right)}\left(\eta_{i} v^{p}\right)^{2 n /(n-2)}\right)^{\frac{n-2}{n}}+\epsilon^{-\frac{a}{1-a}} p^{\frac{2}{1-a}} C^{\frac{1}{1-a}} r_{i}^{-2 \alpha} \oint_{B\left(x, r_{i}\right)} \eta_{i}^{2} v^{2 p},
\end{aligned}
$$


for any $\epsilon>0$ and $a=\frac{n}{2 q}$. Here, the last step above is followed similarly as in (7.7). Therefore, (7.8) becomes

$$
\begin{aligned}
\oint_{B\left(x, r_{i}\right)}\left|\nabla\left(\eta_{i} v^{p}\right)\right|^{2} \leq & \oint_{B\left(x, r_{i}\right)} 8 \lambda p \eta_{i}^{2} v^{2 p}+16 p v^{2 p}\left|\nabla \eta_{i}\right|^{2} \\
& +\epsilon r_{i}^{-2 \alpha}\left(\oint_{B\left(x, r_{i}\right)}\left(\eta_{i} v^{p}\right)^{\frac{2 n}{n-2}}\right)^{\frac{n-2}{n}}+\epsilon^{-\frac{a}{1-a}} C^{\frac{2 q}{2 q-n}} p^{\frac{4 q}{2 q-n}} r_{i}^{-2 \alpha} \oint_{B\left(x, r_{i}\right)} \eta_{i}^{2} v^{2 p} \\
& +70 \epsilon\left(\oint_{B\left(x, r_{i}\right)}\left(\eta_{i} v^{p}\right)^{\frac{2 n}{n-2}}\right)^{\frac{n-2}{n}}+70 \epsilon^{-\frac{a}{1-a}} C^{\frac{2 q}{2 q-n}} p^{\frac{4 q}{2 q-n}} \oint_{B\left(x, r_{i}\right)} \eta_{i}^{2} v^{2 p} .
\end{aligned}
$$

By the Sobolev inequality (3.7) and (7.9),

$$
\begin{aligned}
& \quad\left(\oint_{B\left(x, r_{i}\right)}\left(\eta_{i} v^{p}\right)^{\frac{2 n}{n-2}}\right)^{(n-2) / n} \\
& \leq C(n) r_{i}^{2} \oint_{B\left(x, r_{i}\right)}\left|\nabla\left(\eta_{i} v^{p}\right)\right|^{2} \\
& \leq C(n) r_{i}^{2} \oint_{B\left(x, r_{i}\right)} 8 \lambda p \eta_{i}^{2} v^{2 p}+16 p v^{2 p}\left|\nabla \eta_{i}\right|^{2} \\
& \quad+C(n) \epsilon r_{i}^{2-2 \alpha}\left(\oint_{B}\left(\eta v^{p}\right)^{\frac{2 n}{n-2}}\right)^{\frac{n-2}{n}}+C(n) \epsilon^{-\frac{a}{1-a}} C^{\frac{2 q}{2 q-n}} p^{\frac{4 q}{2 q-n}} r_{i}^{2-2 \alpha} \oint_{B\left(x, r_{i}\right)} \eta_{i}^{2} v^{2 p} \\
& \quad+C(n) r_{i}^{2} \epsilon\left(\oint_{B\left(x, r_{i}\right)}\left(\eta_{i} v^{p}\right)^{\frac{2 n}{n-2}}\right)^{\frac{n-2}{n}}+C(n) r_{i}^{2} \epsilon^{-\frac{a}{1-a}} p^{\frac{4 q}{2 q-n}} \oint_{B\left(x, r_{i}\right)} \eta_{i}^{2} v^{2 p} .
\end{aligned}
$$

Since $r_{i} \leq r \leq 1$ and $\alpha<1$, we may choose $\epsilon=\epsilon(n)$ small so that the above inequality becomes

$$
\left(\oint_{B\left(x, r_{i}\right)}\left(\eta_{i} v^{p}\right)^{\frac{2 n}{n-2}}\right)^{\frac{n-2}{n}} \leq C(n, \lambda, K, \alpha, \rho) r_{i}^{2} \oint_{B\left(x, r_{i}\right)} p v^{2 p}\left|\nabla \eta_{i}\right|^{2}+p^{\frac{4 q}{2 q-n}} \eta_{i}^{2} v^{2 p}
$$

From the volume comparison theorem, we have

$$
\begin{aligned}
\left(\oint_{B\left(x, r_{i+1}\right)}\left(v^{p}\right)^{2 n /(n-2)}\right)^{(n-2) / n} & \leq C(n, \lambda, K, \alpha, \rho)\left(\oint_{B\left(x, r_{i}\right)}\left(\eta_{i} v^{p}\right)^{2 n /(n-2)}\right)^{(n-2) / n} \\
& \leq C(n, \lambda, K, \alpha, \rho) \oint_{B\left(x, r_{i}\right)} 2^{2 i} p v^{2 p}+p^{\frac{4 q}{2 q-n}} v^{2 p}
\end{aligned}
$$

Now let $\mu=\frac{n}{n-2}$ and choose $p=\frac{1}{2} \mu^{i}$ for $i=0,1,2, \cdots$. Then

$$
\begin{aligned}
\left(\oint_{B\left(x, r_{i+1}\right)} v^{\mu^{i+1}}\right)^{(n-2) / n} & =\left(\oint_{B\left(x, r_{i+1}\right)}\left(v^{p}\right)^{2 n /(n-2)}\right)^{(n-2) / n} \\
& \leq C(n, \lambda, K, \alpha, \rho)\left(2^{2 i-1} \mu^{i}+\mu^{2 q i /(2 q-n)}\right) \oint_{B\left(x, r_{i}\right)} v^{\mu^{i}} \\
& \leq C(n, \lambda, K, \alpha, \rho) 4^{2 q i /(2 q-n)} \oint_{B\left(x, r_{i}\right)} v^{\mu^{i}}
\end{aligned}
$$


i.e.,

$$
\|v\|_{\mu^{i+1}, B\left(x, r_{i+1}\right)}^{*} \leq C^{\mu^{-i}} 4^{\frac{2 q}{2 q-n} i \mu^{-i}}\|v\|_{\mu^{i}, B\left(x, r_{i}\right)}^{*}
$$

By using (7.12) iteratively, we get

$$
\sup _{B\left(x, \frac{1}{2} r\right)} v \leq C^{\sum \mu^{-i}} 4^{\frac{2 q}{2 q-n} \sum i \mu^{-i}}\|v\|_{1, B\left(x, \frac{3}{4} r\right)}^{*} \leq C(n, \lambda, K, \alpha, \rho)\|v\|_{1, B\left(x, \frac{3}{4} r\right)}^{*} .
$$

Choose $\eta=\phi(d(x, \cdot))$, where $\phi$ satisfies

$$
\operatorname{supp} \phi \subset[0, r], \phi_{l} \equiv 1 \text { in }\left[0, \frac{3}{4} r\right],\left|\phi^{\prime}\right| \leq 5 r^{-1} \text {. }
$$

Since

$$
\begin{aligned}
\int_{B(x, r)} \eta^{2}|\nabla u|^{2} & =\int_{B(x, r)}-\eta^{2} u f-2 \eta u \nabla_{i} u \nabla_{i} \eta \\
& \leq \int_{B(x, r)} \frac{1}{2} u^{2} \eta^{2}+\frac{1}{2} f^{2} \eta^{2}+\frac{1}{2} \eta^{2}|\nabla u|^{2}+2 u^{2}|\nabla \eta|^{2}
\end{aligned}
$$

this together with the definition of $\eta$ imply that

$$
\begin{aligned}
\oint_{B(x, r)} \eta^{2}|\nabla u|^{2} & \leq 4 \oint_{B(x, r)} u^{2} \eta^{2}+f^{2} \eta^{2}+u^{2}|\nabla \eta|^{2} \\
& \leq 100 r^{-2}\left(\|u\|_{2, B(x, r)}^{*}\right)^{2}+4\left\|f^{2}\right\|_{q, B(x, r)}^{*} .
\end{aligned}
$$

From above, we arrive at

$$
\begin{aligned}
\|v\|_{1, B\left(x, \frac{3}{4} r\right)}^{*} & \leq \frac{\operatorname{Vol}(B(x, r))}{\operatorname{Vol}\left(B\left(x, \frac{3}{4} r\right)\right)} \oint_{B(x, r)} \eta^{2}\left(|\nabla u|^{2}+\left\|f^{2}\right\|_{q, B(x, r)}^{*}\right) \\
& \leq C(n, \lambda, K, \alpha, \rho) r^{-2}\left[\left(\|u\|_{2, B(x, r)}^{*}\right)^{2}+\left(\|f\|_{2 q, B(x, r)}^{*}\right)^{2}\right] .
\end{aligned}
$$

Combining (7.13) and (7.14), we deduce that, for any $q \in\left(n, \frac{n}{\alpha}\right)$,

$$
\sup _{B\left(x, \frac{1}{2} r\right)}|\nabla u|^{2} \leq\|v\|_{\infty, B\left(x, \frac{1}{2} r\right)} \leq C(n, \lambda, K, \alpha, \rho) r^{-2}\left[\left(\|u\|_{2, B(x, r)}^{*}\right)^{2}+\left(\|f\|_{2 q, B(x, r)}^{*}\right)^{2}\right] .
$$

This finishes the proof of the theorem due to the fact that $\|f\|_{q, B(x, r)}^{*} \leq\|f\|_{q^{\prime}, B(x, r)}^{*}$ whenever $q^{\prime} \geq q$.

If $\alpha=0$, then the Sobolev inequality in section 3 holds without the volume noncollapsing condition. Therefore the conclusion of the theorem also holds without it.

\section{ApPENDIX II}

Here we give a proof of Theorem 5.1, the segment inequality.

Proof of Theorem 5.1. We may assume that the minimal geodesic from $x_{1}$ to $x_{2}$ is unique, since the set of this type of points $\left(x_{1}, x_{2}\right)$ is dense. Moreover, since

$$
\begin{aligned}
\mathcal{F}_{f}\left(x_{1}, x_{2}\right) & =\int_{0}^{\frac{d\left(x_{1}, x_{2}\right)}{2}} f(\gamma(s)) d s+\int_{\frac{d\left(x_{1}, x_{2}\right)}{2}}^{d\left(x_{1}, x_{2}\right)} f(\gamma(s)) d s \\
& =\int_{\frac{d\left(x_{1}, x_{2}\right)}{2}}^{d\left(x_{1}, x_{2}\right)} f\left(\gamma\left(d\left(x_{1}, x_{2}\right)-t\right)\right) d t+\int_{\frac{d\left(x_{1}, x_{2}\right)}{2}}^{d\left(x_{1}, x_{2}\right)} f(\gamma(s)) d s \\
& :=\mathcal{F}_{f, 1}\left(x_{1}, x_{2}\right)+\mathcal{F}_{f, 2}\left(x_{1}, x_{2}\right),
\end{aligned}
$$


to estimate $\mathcal{F}_{f}$ it suffices to estimate $\mathcal{F}_{f, 2}\left(x_{1}, x_{2}\right)$.

Now fixe $x_{1} \in A_{1}$. For any unit tangent vector $\theta$ at $x_{1}$, let $\gamma(0)=x_{1}$ and $\gamma^{\prime}(0)=\theta$. Let $l(\theta)$ be the largest number $\leq 2 r$ such that $\left.\gamma\right|_{[0, l(\theta)]}$ is minimal. Then

$$
\begin{aligned}
\int_{A_{2}} \mathcal{F}_{f, 2}\left(x_{1}, x_{2}\right) d g\left(x_{2}\right) & \leq \int_{B\left(x_{1}, 2 r\right)} \mathcal{F}_{f, 2}\left(x_{1}, x_{2}\right) d g\left(x_{2}\right) \\
& =\int_{S^{n-1}} \int_{0}^{l(\theta)} \mathcal{F}_{f, 2}\left(x_{1}, \gamma(s)\right) w(s, \theta) d s d \theta \\
& =\int_{S^{n-1}} \int_{0}^{l(\theta)}\left[\int_{\frac{s}{2}}^{s} f(\gamma(u)) d u\right] w(s, \theta) d s d \theta \\
& \leq \int_{S^{n-1}} \int_{0}^{l(\theta)} \int_{\frac{s}{2}}^{s} f(\gamma(u)) w(u, \theta) C(n, \lambda, K, \alpha) \frac{s^{n-1}}{u^{n-1}} d u d s d \theta \\
& \leq C(n, \lambda, K, \alpha) \int_{S^{n-1}} \int_{0}^{2 r} \int_{0}^{2 r} f(\gamma(u)) w(u, \theta) d u d s d \theta \\
& =C(n, \lambda, K, \alpha) r \int_{B\left(x_{1}, 2 r\right)} f(x) d g \\
& \leq C(n, \lambda, K, \alpha) r \int_{B(x, 3 r)} f(x) d g .
\end{aligned}
$$

In the fourth step above, we have used (2.14) to get

$$
w(s, \theta) \leq C(n, \lambda, K, \alpha) \frac{s^{n-1}}{u^{n-1}} w(u, \theta) .
$$

Thus,

$$
\int_{A_{1}} \int_{A_{2}} \mathcal{F}_{f, 2}\left(x_{1}, x_{2}\right) d g\left(x_{2}\right) d g\left(x_{1}\right) \leq C(n, \lambda, K, \alpha) r \operatorname{Vol}\left(A_{1}\right) \int_{B(x, 3 r)} f(x) d g .
$$

Similarly, we can prove

$$
\int_{A_{2}} \int_{A_{1}} \mathcal{F}_{f, 1}\left(x_{1}, x_{2}\right) d g\left(x_{1}\right) d g\left(x_{2}\right) \leq C(n, \lambda, K, \alpha) r \operatorname{Vol}\left(A_{2}\right) \int_{B(x, 3 r)} f(x) d g .
$$

This finishes the proof of the theorem.

Next, we prove Lemma 6.4. First from Theorem 2.2, we can make the following Claim. Suppose that (5.1) and (5.2) hold. Let $r \leq 1$, and $0<\eta<1$. Then for some $\Psi=\Psi(\lambda, K \mid n, \alpha, \rho)$, we have

$$
\frac{\operatorname{Vol}(\partial B(x, r))}{\operatorname{Vol}(\partial \underline{B}(0, r))} \leq(1+\Psi) \frac{\operatorname{Vol}\left(A_{\eta r, r}(x)\right)}{\operatorname{Vol}\left(\underline{A}_{\eta r, r}(0)\right)},
$$

and

$$
\frac{\operatorname{Vol}\left(A_{\eta r, r}(x)\right)}{\operatorname{Vol}\left(\underline{A}_{\eta r, r}(0)\right)} \leq(1+\Psi) \frac{\operatorname{Vol}(\partial B(x, \eta r))}{\operatorname{Vol}(\partial \underline{B}(0, \eta r))} .
$$

Here $A_{r_{1}, r_{2}}(x)=B\left(x, r_{2}\right)-B\left(x, r_{1}\right)$ is the annulus in $\mathbf{M}$ and $\underline{A}_{r_{1}, r_{2}}(0)$ is the corresponding Euclidean annulus. 
Proof. Given $r \leq 1$, for any $s<r$, by Theorem 2.2 , we have

$$
\frac{w(r, \cdot)}{r^{n-1}} \leq(1+\Psi) \frac{w(s, \cdot)}{s^{n-1}} .
$$

It follows that

$$
s^{n-1} \int_{S^{n-1}} w(r, \theta) d \theta \leq(1+\Psi) r^{n-1} \int_{S^{n-1}} w(s, \theta) d \theta .
$$

Integrating both sides from $\eta r$ to $r$ with respect to $s$ gives (8.1).

Similarly, one can show (8.2). This proves the claim.

Now we are ready to prove Lemma 6.4, which is

Lemma 8.1. If

$$
\left(1-\delta^{\prime}\right) \frac{\operatorname{Vol}\left(B\left(x, \frac{1}{2} R\right)\right)}{\operatorname{Vol}\left(\underline{B}\left(0, \frac{1}{2} R\right)\right)} \leq \frac{\operatorname{Vol}(B(x, R))}{\operatorname{Vol}(\underline{B}(0, R))},
$$

then for any $0<\eta \leq \frac{1}{2}$ and some $\Psi=\Psi\left(\delta^{\prime}, \lambda, K \mid n, \alpha, \rho\right)$, we have

$$
(1-\Psi) \frac{\operatorname{Vol}(B(x,(1-\eta) R))}{\operatorname{Vol}(\underline{B}(0,(1-\eta) R))} \leq \frac{\operatorname{Vol}(\partial B(x,(1-\eta) R))}{\operatorname{Vol}(\partial \underline{B}(0,(1-\eta) R))} .
$$

Proof. By Theorem 2.2 and the assumption of the lemma, we have

$$
\left(1-\delta^{\prime}\right) \frac{\operatorname{Vol}(B(x,(1-\eta) R))}{\operatorname{Vol}(\underline{B}(0,(1-\eta) R))} \leq\left(1-\delta^{\prime}\right)(1+\Psi) \frac{\operatorname{Vol}\left(B\left(x, \frac{1}{2} R\right)\right)}{\operatorname{Vol}\left(\underline{B}\left(0, \frac{1}{2} R\right)\right)} \leq(1+\Psi) \frac{\operatorname{Vol}(B(x, R))}{\operatorname{Vol}(\underline{B}(0, R))} .
$$

It implies that

$$
\left(1-\delta^{\prime}\right) \operatorname{Vol}(B(x,(1-\eta) R)) \operatorname{Vol}(\underline{B}(0, R)) \leq(1+\Psi) \operatorname{Vol}(B(x, R)) \operatorname{Vol}(\underline{B}(0,(1-\eta) R)) .
$$

Subtracting $\left(1-\delta^{\prime}\right) \operatorname{Vol}(B(x,(1-\eta) R)) \operatorname{Vol}(\underline{B}(0,(1-\eta) R))$ from both sides above yields

$$
\begin{aligned}
& \left(1-\delta^{\prime}\right) \operatorname{Vol}(B(x,(1-\eta) R)) \operatorname{Vol}\left(\underline{A}_{(1-\eta) R, R}(0)\right) \\
\leq & \operatorname{Vol}\left(A_{(1-\eta) R, R}(x)\right) \operatorname{Vol}(\underline{B}(0,(1-\eta) R))+\Psi \operatorname{Vol}(B(x, R)) \operatorname{Vol}(\underline{B}(0,(1-\eta) R)) \\
& +\delta^{\prime} \operatorname{Vol}(B(x,(1-\eta) R)) \operatorname{Vol}(\underline{B}(0,(1-\eta) R)) .
\end{aligned}
$$

Dividing both sides above by $\operatorname{Vol}(\underline{B}(0,(1-\eta) R)) \operatorname{Vol}\left(\underline{A}_{(1-\eta) R, R}(0)\right)$ and using Theorem 2.2 give

$$
\begin{aligned}
& \left(1-\delta^{\prime}\right) \frac{\operatorname{Vol}(B(x,(1-\eta) R))}{\operatorname{Vol}(\underline{B}(0,(1-\eta) R))} \\
\leq & \frac{\operatorname{Vol}\left(A_{(1-\eta) R, R}(x)\right)}{\operatorname{Vol}\left(\underline{A}_{(1-\eta) R, R}(0)\right)}+\Psi \frac{\operatorname{Vol}(B(x, R))}{\operatorname{Vol}\left(\underline{A}_{(1-\eta) R, R}(0)\right)}+\delta^{\prime} \frac{\operatorname{Vol}(B(x,(1-\eta) R))}{\operatorname{Vol}\left(\underline{A}_{(1-\eta) R, R}(0)\right)} \\
\leq & \frac{\operatorname{Vol}\left(A_{(1-\eta) R, R}(x)\right)}{\operatorname{Vol}\left(\underline{A}_{(1-\eta) R, R}(0)\right)}+\left[2^{n} \Psi(1+\Psi)+\delta^{\prime}\right] \frac{\operatorname{Vol}(B(x,(1-\eta) R))}{\operatorname{Vol}\left(\underline{A}_{(1-\eta) R, R}(0)\right)} .
\end{aligned}
$$

Moving the second term on the LHS to the RHS and using the above claim, we get

$$
(1-\delta) \frac{\operatorname{Vol}(B(x,(1-\eta) R))}{\operatorname{Vol}(\underline{B}(0,(1-\eta) R))} \leq(1+\Psi) \frac{\operatorname{Vol}(\partial B(x,(1-\eta) R))}{\operatorname{Vol}(\partial \underline{B}(0,(1-\eta) R))}
$$

where

$$
\delta=\delta^{\prime}+\left[2^{n} \Psi(1+\Psi)+\delta^{\prime}\right] \frac{\operatorname{Vol}(\underline{B}(0,(1-\eta) R))}{\operatorname{Vol}\left(\underline{A}_{(1-\eta) R, R}(0)\right)} .
$$

This finishes the proof of the lemma. 


\section{ACKNOWLEDGEMENTS}

Q.S.Z is grateful to the Simons foundation for its support. M. Z would like to thank Prof. Huai-Dong Cao for constant support and his interest in this paper. M. Z's research is partially supported by NSFC Grant No. 11501206. Both authors wish to thank Professors G.F. Wei, Z.L. Zhang and X.H. Zhu for helpful suggestions.

\section{REFERENCES}

[AG] Abresch, Uwe; Gromoll, Detlef, On complete manifolds with nonnegative Ricci curvature. J. Amer. Math. Soc. 3 (1990), no. 2, 355-374.

[BE] Bakry, Dominique; Émery, Michel, Diffusions hypercontractives. (French) [Hypercontractive diffusions] S eminaire de probabilit es, XIX, 1983/84, 177-206, Lecture Notes in Math., 1123, Springer, Berlin, 1985.

[BQ] Bakry, Dominique; Qian, Zhongmin, Volume comparison theorems without Jacobi fields. Current trends in potential theory, 115-122, Theta Ser. Adv. Math., 4, Theta, Bucharest, 2005.

[Bam] Bamler, Richard, Compactness properties of Ricci flows with bounded scalar curvature, arXiv:1512.08527,

[BZ] Bamler, Richard; Qi S. Zhang, Heat kernel and curvature bounds in Ricci flows with bounded scalar curvature, arXiv: 1501.01291 ,

[Cao1] Cao, Huai-Dong, Recent progress on Ricci solitons. Recent advances in geometric analysis, 1-38, Adv. Lect. Math. (ALM), 11, Int. Press, Somerville, MA, 2010.

[Cao2] Cao, Huai-Dong, Geometry of complete gradient shrinking Ricci solitons. Geometry and analysis. No. 1, 227-246, Adv. Lect. Math. (ALM), 17, Int. Press, Somerville, MA, 2011.

[CZ] Cao, Huai-Dong; Zhou, Detang, On complete gradient shrinking Ricci solitons. J. Differential Geom. 85 (2010), no. 2, 175-185

[Ch] Cheeger, Jeff, Degeneration of Riemannian metrics under Ricci curvature bounds, Lezioni Fermiane. [Fermi Lectures] Scuola Normale Superiore, Pisa, 2001.

[ChCo1] Cheeger, Jeff; Colding, Tobias H., Lower bounds on Ricci curvature and the almost rigidity of warped products. Ann. of Math. (2) 144 (1996), no. 1, 189-237.

[ChCo2] Cheeger, Jeff; Colding, Tobias H., On the structure of spaces with Ricci curvature bounded below. I. J. Differential Geom. 46 (1997), no. 3, 406-480.

[ChNa] Cheeger, Jeff; Naber, Aaron, Regularity of Einstein Manifolds and the codimension 4 Conjecture, Ann. Math. 182 (2015), 1093-1165.

[Co] Colding, Tobias H., Ricci curvature and volume convergence. Ann. of Math. (2) 145 (1997), no. $3,477-501$.

[CN] Colding, Tobias Holck; Naber, Aaron, Sharp Hölder continuity of tangent cones for spaces with a lower Ricci curvature bound and applications. Ann. of Math. (2) 176 (2012), no. 2, 1173-1229.

[Chen] Chen, Bing-Long, Strong uniqueness of the Ricci flow. J. Differential Geom. 82 (2009), no. 2, 363-382.

[D] Derdzinski, Andrzej, A Myers-type theorem and compact Ricci solitons. Proc. Amer. Math. Soc. 134 (2006), no. 12, 3645-3648

[DWZ] Dai, Xianzhe; Wei, Guofang; Zhang, Zhenlei, Local Sobolev Constant Estimate for Integral Ricci Curvature Bounds, arXiv:1601.08191

[FG] Fernández-López, M.; Garcia-Rio, E. A remark on compact Ricci solitons. Math. Ann. 340 (2008), no. 4, 893-896.

[Gro] Gromov, M., Paul Lévy's isoperimetric inequality, Inst. Hautes Etudes Sci. (1980), preprint. http://www.ihes.fr/ gromov/PDF/11[33].pdf

[GT] Gilbarg, David; Trudinger, Neil S. Elliptic partial differential equations of second order. Grundlehren der Mathematischen Wissenschaften, Vol. 224. Springer-Verlag, Berlin-New York, 1977. $\mathrm{x}+401 \mathrm{pp}$.

[xLi] Li, Xiang-Dong, Liouville theorems for symmetric diffusion operators on complete Riemannian manifolds. J. Math. Pures Appl. (9) 84 (2005), no. 10, 1295-1361. 
[Pe] Perelman, G. Manifolds of positive Ricci curvature with almost maximal volume. J. Amer. Math. Soc. 7 (1994), no. 2, 299-305.

[PeWe1] Petersen, Peter; Wei, Guofang, Relative volume comparison with integral curvature bounds. Geom. Funct. Anal. 7 (1997), no. 6, 1031-1045.

[PeWe2] Petersen, Peter; Wei, Guofang, Analysis and geometry on manifolds with integral Ricci curvature bounds. II, Trans. Amer. Math. Soc., 353 (2001), 457-478.

[Q] Qian, Zhongmin, A comparison theorem for an elliptic operator. Potential Anal. 8 (1998), no. 2, 137-142.

[Re] Reifenberg, E. R. Solution of the Plateau Problem for m-dimensional surfaces of varying topological type. Acta Math. 1041960 1-92.

[TZ] Tian, Gang; Zhang, Zhenlei, Degeneration of Kähler-Ricci solitons. Int. Math. Res. Not. IMRN 2012, no. 5, 957-985.

[TZ2] Tian, Gang; Zhang, Zhenlei, Regularity of Khler-Ricci flows on Fano manifolds, arXiv:1310.5897.

[W] Wylie, William, Complete shrinking Ricci solitons have finite fundamental group. Proc. Amer. Math. Soc. 136 (2008), no. 5, 1803-1806.

[WW] Wei, Guofang; Wylie, William, Comparison geometry for the Bakry-Émery Ricci tensor. J. Differential Geom. 83 (2009), no. 2, 377-405.

[WZ] Wang, Feng; Zhu, Xiaohua, Structure of spaces with Bakry-Émery Ricci curvature bounded below, arXiv:1304.4490

[ZZ] Zhang, Qi S.; Zhu, Meng, Li-Yau gradient bounds under nearly optimal curvature conditions, arXiv:1511.00791.

[zlZh] Zhang, Zhenlei, Degeneration of shrinking Ricci solitons. Int. Math. Res. Not. IMRN 2010 , no. $21,4137-4158$.

[zZh] Zhang, Zhu-Hong, On the completeness of gradient Ricci solitons. Proc. Amer. Math. Soc. 137 (2009), no. 8, 2755-2759.

Department of Mathematics, University of California, Riverside, Riverside, CA 92521, USA

E-mail address: qizhang@math.ucr.edu

Department of Mathematics, East China Normal University, Shanghai 200241, China - And Department of Mathematics, University of California, Riverside, Riverside, CA 92521, USA

E-mail address: mzhu@math.ucr.edu 